\title{
Determination of the Uncertainties in S-Curve Logistic Fits
}

\author{
A. DEBECKER AND T. MODIS
}

\section{ABSTRACT}

Look-up tables and graphs are provided for determining the uncertainties during logistic fits, on the three parameters $M, \alpha$ and $t_{0}$ describing an S-curve of the form:

$$
\mathrm{S}(\mathrm{t})=\frac{\mathrm{M}}{1+e^{-\mathrm{a}\left(t-t_{0}\right)}}
$$

The uncertainties and the associated confidence levels are given as a function of the uncertainty on the data points and the length of the historical period. Correlations between these variables are also examined; they make "what-if" games possible even before doing the fit.

The study is based on some $35,000 \mathrm{~S}$-curve fits on simulated data covering a variety of conditions and carried out via a $\chi^{2}$ minimization technique. A rule-of-thumb general result is that, given at least half of the S-curve range and a precision of better than $10 \%$ on each hitorical point, the uncertainty on $\mathrm{M}$ will be less than $20 \%$ with $90 \%$ confidence level.

\section{Introduction}

S-curve logistic fitting has been successful in describing learning and/or growing processes. A variety of applications ranging from biology (echo-niche filling of species) to art and industry (market-niche filling of products) abounds in the literature [1-4].

The most facinating aspect of S-curve fitting is the ability to predict from early measurements the final maximum, a fact that often shocks and sometimes vexes individuals, with its inherent element of predeterminism. This very fact, however, constitutes also the fundamental weakness and the major criticism in S-curve fitting, namely the uncertainty involved in an early determination of the final maximum. A three-parameter logistic fit can sometimes accomodate wildly different values for the final maximum. Obviously, the more precise the data and the more of the S-curve range they cover, the more accurate the determination of the final maximum but, unfortunately, at the same time, the less interesting this determination becomes.

The need for quantitative determination of the uncertainties resulting from such fits has not been adequately addressed up to now. In this work a study was undertaken to quantify the uncertainties on the parameters determined by logistic S-curve fits.

THEODORE MODIS is a physicist and a senior management strategy consultant at Digital Equipment Co. He has taught at Columbia University, New York, NY; the University of Geneva, Geneva, Switzerland; INSEAD, Fontainebleau, France; and IMD, Lausanne, Switzerland. ALAIN DEBECKER is a mathematician and a management science consultant, teaching Quantitative Methods for management at Lyon University. 
In the next (second) section the logistic equation itself is described and the adopted approach justified. In the third section the generation of the simulated data and the fitting procedure are given. The fourth section gives the results in the form of look-up tables and figures. Conclusions are presented in the final section.

\section{Logistic Growth}

Growth in a biological context has been described successfully by the Voltera differential equation [5]:

$$
\dot{q}(t)=\frac{\alpha}{\mathrm{M}} q(t)(\mathrm{M}-q(t))
$$

where $\alpha$ and $\mathrm{M}$ are constants characterizing the rate of growth and the final size respectively.

The solution of this equation gives a typical S-curve

$$
q(t)=\frac{M}{1+e^{-\alpha\left(t-t_{0}\right)}}
$$

where $t_{o}$ is an integration constant localizing the process in time.

Now given a set of date $\left\{\left(t_{i}, q_{i}\right) \mid i=1, \ldots, \mathrm{n}\right\}$ and considering $q_{i}$ as one observation of the discrete random variable $Q\left(t_{i}\right)$, the quantity

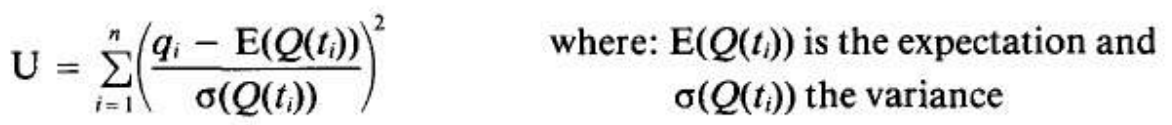

yields a $\chi^{2}$ distribution if $Q\left(t_{i}\right)$ is normally distributed for each $i$.

To fit the data points to a certain analytic form we must define the expected values $\mathrm{E}(\mathrm{Q}(\mathrm{t}))$ of the random discrete variable $\mathrm{Q}(\mathrm{t})$ according to the law in question and then the variance $\sigma(Q(t)$ around these expected values. It is shown in the appendix that for a logistic S-curve, $\mathrm{Q}(t)$ obeys a binominal law $\mathbb{B}\left(\mathrm{M}_{2}\right)$, where

$$
f=\frac{1}{1+e^{-\alpha\left(t-t_{o}\right)}}
$$

and that its expectation and variance are:

$$
\begin{aligned}
& \mathrm{E}(\mathrm{Q}(t))=q(t)=\frac{\mathrm{M}}{1+e^{-\mathrm{a}\left(t-t_{0}\right)}} \\
& \sigma^{2}(\mathrm{Q}(\mathrm{E}))=\mathrm{M} f(1-f)=\frac{\mathrm{M}}{\left(1+e^{-\alpha\left(t-t_{0}\right)}\right)\left(1+e^{\alpha\left(t-t_{0}\right)}\right)}
\end{aligned}
$$

To the extent that $\mathrm{M}$ is large and $\mathrm{O} \ll q(t) \ll \mathrm{M}, \mathrm{Q}(t)$ will be normally distributed and (2) will approximate a $\chi^{2}$ distribution with $n-3$ degrees of freedom. This condition is reflected in the usually applied rule-of-thumb where normal distributions are assumed for

$$
0.1<\frac{q(t)}{\mathrm{M}}<0.9
$$

In this range, then, minimization of (2), i.e., setting its gradient to zero, will determine the values of the three parameters $\mathrm{M}, \alpha$ and $t_{o}$. At the same time the matrix of the second partial derivatives allows, in principle, the determination of the standard deviations errors - on the values of the parameters and the corresponding confidence levels. 

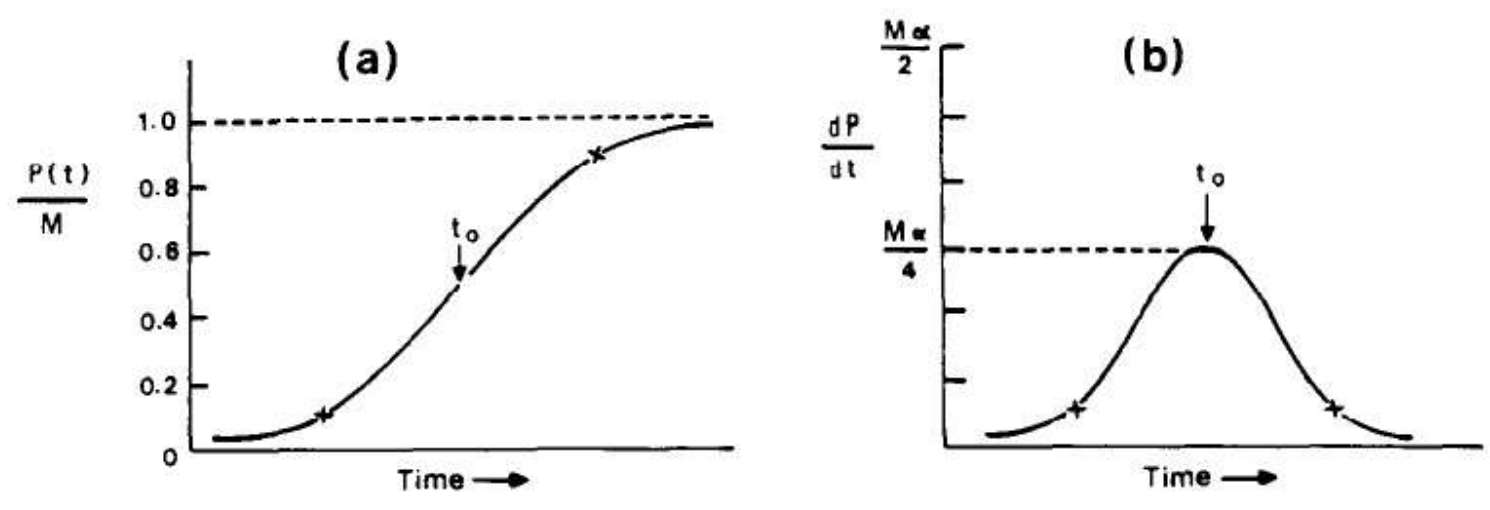

Fig. 1. (a) S-curve typical of population growth. (b) Time derivative of (a) typical of life cycle.

However, in our case, determination of confidence levels and errors by the above method is not suitable. It implies exceedingly complicated calculations and it is likely to give biased results in the case where the three parametcrs are corrclated between them in a nonlinear way. Furthermore, it is not applicable to the extent that the three parameters are not normally distributed.

Therefore, a numerical approach was adopted for the determination of the confidence levels and uncertainties involved in the parameter values found by the $\chi^{2}$ minimization. A large number of fits were carried out on simulated data statistically deviated around the theoretical value and covering a variety of time spans. Distribution for the values of the three parameters $\mathrm{M}, \alpha$ and $t_{o}$ were obtained through a $\chi^{2}$ minimization, and comparison with the theoretical values used in generating the data, provided a means for establishing uncertainties, confidence levels, systematic biases (if any) and correlations between the three parameters.

An a posteriori justification for adopting the numerical approach can be found in Figure 7 where indeed strong nonlinear correlations are witnessed, and in Figure 3 where deviations from normal distributions are evident.

\section{Generation of Simulated Data and Fitting Procedure}

An S-curve, Figure 1(a), represents the cumulated growth as a function of time, e.g., the population of a species at time $t$ or the total number of units of a certain model produced by a manufacturer up to time $t$, etc. The data, however, are most frequently available in terms of the rate of growth, the time derivative of the S-curve, Figure 1(b); typical examples are reproduction rates, productivities, units sold per trimester, etc.; in other words, life cycles.

The simulation data were therefore generated according to the time derivative of equation (1), namely

$$
q\left(t_{i}\right)=\frac{\mathrm{M}}{\left(1+e^{-\alpha\left(t-t_{o}\right)}\right)\left(1+e^{\alpha\left(t-t_{o}\right)}\right)}
$$

where, without loss of generality here, we take $\mathrm{M}=1, \alpha=1$ and $t_{o}=0, i=1$ to 20 , defining 20 equal time bins $t_{i}$. The time span $t_{1}-t_{20}$ was chosen such that it covered a certain portion of the complete S-curve. Nine distinct cases were considered, namely:

$q\left(t_{i}\right)$ in the range of $1 \%$ to $20 \%$ of $\mathrm{M}$

$q\left(t_{i}\right)$ in the range of $1 \%$ to $30 \%$ of $M$ 
TABLE 1

Expected uncertainties on fitted parameters for the range $1 \%-20 \%$ as a function of confidence level (vertically) and error on historical data (horizontally).

\begin{tabular}{|c|c|c|c|c|c|c|}
\hline Parameter: M & 1 & 5 & 10 & 15 & 20 & 25 \\
\hline 70 & 4.9 & 22 & 51 & 120 & 190 & 290 \\
\hline 75 & 5.9 & 27 & 60 & 150 & 230 & 360 \\
\hline 80 & 6.8 & 32 & 72 & 180 & 280 & 430 \\
\hline 85 & 8.6 & 37 & 92 & 250 & 360 & 480 \\
\hline 90 & 11 & 48 & 120 & 300 & 440 & 720 \\
\hline 95 & 15 & 65 & 160 & 480 & 640 & \\
\hline 99 & 65 & & & & & \\
\hline Parameter: $\alpha$ & 1 & 5 & 10 & 15 & 20 & 25 \\
\hline 70 & 0.5 & 2.6 & 5.2 & 7.5 & 9.0 & 12 \\
\hline 75 & 0.6 & 3.0 & 5.8 & 8.2 & 11 & 13 \\
\hline 80 & 0.8 & 3.5 & 6.5 & 9.5 & 11 & 15 \\
\hline 85 & 0.9 & 3.9 & 7.1 & 10 & 13 & 16 \\
\hline 90 & 1.1 & 4.5 & 8.1 & 11 & 14 & 19 \\
\hline 95 & 1.7 & 5.2 & 9.2 & 13 & 17 & 21 \\
\hline 99 & 3.5 & 7.2 & 11 & 16 & 21 & 28 \\
\hline Parameter: $t_{v}$ & 1 & 5 & 10 & 15 & 20 & 25 \\
\hline 70 & 0.064 & 0.30 & 0.61 & 1.1 & 1.3 & 1.7 \\
\hline 75 & 0.808 & 0.35 & 0.70 & 1.1 & 1.5 & 1.8 \\
\hline 80 & 0.089 & 0.40 & 0.80 & 1.3 & 1.6 & 2.0 \\
\hline 85 & 0.11 & 0.46 & 0.90 & 1.5 & 1.8 & 2.1 \\
\hline 90 & 0.15 & 0.53 & 1.1 & 1.7 & 2.0 & 2.4 \\
\hline 95 & 0.20 & 0.70 & 1.3 & 1.9 & 2.2 & 2.6 \\
\hline 99 & 0.60 & 2.3 & 1.6 & 3.4 & 2.9 & 3.2 \\
\hline
\end{tabular}

All numbers are in $\%$ except the uncertainties on $t_{o}$; see text.

TABLE 2

Expected uncertainties on fitted parameters for the range $1 \%-30 \%$ as a function of confidence level (vertically) and error on historical data (horizontally).

\begin{tabular}{ccccccc}
\hline Parameter: $\mathbf{M}$ & 1 & 5 & 10 & 15 & 20 & 25 \\
\hline 70 & 2.7 & 13 & 28 & 47 & 69 & 120 \\
75 & 3.2 & 15 & 32 & 53 & 81 & 190 \\
80 & 3.9 & 17 & 36 & 62 & 110 & 240 \\
85 & 4.8 & 19 & 41 & 71 & 130 & 370 \\
90 & 5.9 & 22 & 48 & 110 & 210 & 470 \\
95 & 8.5 & 29 & 66 & 140 & 350 & 820 \\
99 & 48 & 49 & 180 & 350 & 690 & \\
\hline Parameter: $\alpha$ & 1 & 5 & 10 & 15 & 20 & 25 \\
\hline 70 & 0.4 & 1.9 & 4.5 & 6.3 & 8.1 & 9.9 \\
75 & 0.5 & 2.2 & 5.0 & 6.8 & 8.8 & 11 \\
80 & 0.6 & 2.6 & 5.7 & 7.4 & 9.9 & 12 \\
85 & 0.7 & 2.9 & 6.0 & 8.1 & 11 & 13 \\
90 & 1.1 & 3.4 & 6.7 & 9.4 & 12 & 15 \\
95 & 1.3 & 4.0 & 8.3 & 11 & 15 & 17 \\
99 & 3.2 & 5.6 & 10 & 16 & 19 & 24 \\
\hline Parameter: $t_{o}$ & 1 & 5 & 10 & 15 & 20 & 25 \\
\hline 70 & 0.041 & 0.18 & 0.39 & 0.57 & 0.80 & 1.1 \\
75 & 0.048 & 0.21 & 0.43 & 0.65 & 0.89 & 1.3 \\
80 & 0.057 & 0.24 & 0.49 & 0.73 & 1.0 & 1.5 \\
85 & 0.067 & 0.27 & 0.56 & 0.88 & 1.2 & 1.8 \\
90 & 0.081 & 0.32 & 0.64 & 0.99 & 1.5 & 2.0 \\
95 & 0.12 & 0.39 & 0.77 & 1.2 & 1.8 & 2.3 \\
99 & 0.45 & 0.62 & 1.3 & 1.9 & 2.2 & 3.0 \\
\hline
\end{tabular}

All numbers are in $\%$ except the uncertainties on $t_{p}$; see text. 
TABLE 3

Expected uncertainties on fitted parameters for the range $1 \%-40 \%$ as a function of confidence level (vertically) and error on historical data (horizontally).

\begin{tabular}{|c|c|c|c|c|c|c|}
\hline Parameter: $\mathbf{M}$ & 1 & 5 & 10 & 15 & 20 & 25 \\
\hline 70 & 1.9 & 7.8 & 16 & 26 & 39 & 54 \\
\hline 75 & 2.2 & 8.5 & 18 & 29 & 46 & 63 \\
\hline 80 & 2.5 & 9.7 & 20 & 34 & 53 & 86 \\
\hline 85 & 2.9 & 11 & 23 & 42 & 61 & 110 \\
\hline 90 & 3.6 & 13 & 28 & 50 & 77 & 140 \\
\hline 95 & 5.0 & 17 & 35 & 61 & 150 & 210 \\
\hline 99 & 6.5 & 22 & 55 & 140 & 330 & 470 \\
\hline Parameter: $\boldsymbol{\alpha}$ & 1 & 5 & 10 & 15 & 20 & 25 \\
\hline 70 & 0.4 & 1.7 & 3.5 & 5.3 & 7.3 & 9.7 \\
\hline 75 & 0.4 & 2.0 & 3.9 & 6.0 & 7.7 & 11 \\
\hline 80 & 0.4 & 2.1 & 4.6 & 6.8 & 8.5 & 12 \\
\hline 85 & 0.6 & 2.4 & 5.1 & 7.5 & 9.5 & 13 \\
\hline 90 & 0.7 & 2.9 & 5.8 & 8.4 & 11 & 15 \\
\hline 95 & 1.1 & 3.5 & 7.0 & 9.9 & 13 & 17 \\
\hline 99 & 1.4 & 5.4 & 8.4 & 14 & 18 & 21 \\
\hline Parameter: $t_{o}$ & 1 & 5 & 10 & 15 & 20 & 25 \\
\hline 70 & 0.030 & 0.12 & 0.25 & 0.39 & 0.54 & 0.71 \\
\hline 75 & 0.036 & 0.14 & 0.27 & 0.43 & 0.62 & 0.80 \\
\hline 80 & 0.041 & 0.15 & 0.31 & 0.50 & 0.68 & 0.99 \\
\hline 85 & 0.045 & 0.17 & 0.27 & 0.57 & 0.76 & 1.1 \\
\hline 90 & 0.055 & 0.20 & 0.43 & 0.66 & 0.91 & 1.3 \\
\hline 95 & 0.079 & 0.26 & 0.51 & 0.80 & 1.3 & 1.6 \\
\hline 99 & 0.10 & 0.37 & 0.70 & 1.3 & 1.9 & 2.2 \\
\hline
\end{tabular}

All numbers are in $\%$ except the uncertainties on $t_{o}$; see text.

TABLE 4

Expected uncertainties on fitted parameters for the range $1 \%-50 \%$ as a function of confidence level (vertically) and error on historical data (horizontally).

\begin{tabular}{|c|c|c|c|c|c|c|}
\hline Parameter: M & 1 & 5 & 10 & 15 & 20 & 25 \\
\hline 70 & 1.2 & 5.1 & 11 & 17 & 23 & 29 \\
\hline 75 & 1.4 & 5.5 & 12 & 19 & 26 & 32 \\
\hline 80 & 1.8 & 6.4 & 14 & 22 & 29 & 36 \\
\hline 85 & 2.1 & 7.3 & 16 & 25 & 36 & 42 \\
\hline 90 & 2.6 & 8.8 & 18 & 29 & 42 & 48 \\
\hline 95 & 3.1 & 11 & 21 & 39 & 56 & 66 \\
\hline 99 & 4.6 & 22 & 30 & 55 & 150 & 110 \\
\hline Parameter: $\alpha$ & 1 & 5 & 10 & 15 & 20 & 25 \\
\hline 70 & 0.4 & 1.6 & 3.2 & $5 / 2$ & 6.3 & 7.9 \\
\hline 75 & 0.4 & 1.7 & 3.7 & 6.0 & 7.1 & 8.8 \\
\hline 80 & 0.5 & 1.9 & 4.2 & 6.8 & 7.7 & 9.8 \\
\hline 85 & 0.7 & 2.3 & 4.7 & 7.5 & 8.6 & 11 \\
\hline 90 & 0.7 & 2.6 & 5.4 & 8.4 & 9.9 & 12 \\
\hline 95 & 0.9 & 3.3 & 6.2 & 9.9 & 12 & 14 \\
\hline 99 & 1.4 & 5.4 & 8.3 & 13 & 16 & 21 \\
\hline Parameter: $t_{0}$ & 1 & 5 & 10 & 15 & 20 & 25 \\
\hline 70 & 0.022 & 0.088 & 0.20 & 0.28 & 0.38 & 0.45 \\
\hline 75 & 0.026 & 0.10 & 0.21 & 0.33 & 0.44 & 0.50 \\
\hline 80 & 0.030 & 0.11 & 0.24 & 0.36 & 0.49 & 0.55 \\
\hline 85 & 0.036 & 0.13 & 0.27 & 0.41 & 0.55 & 0.65 \\
\hline 90 & 0.044 & 0.15 & 0.30 & 0.48 & 0.65 & 0.73 \\
\hline 95 & 0.058 & 0.19 & 0.35 & 0.62 & 0.79 & 0.89 \\
\hline 99 & 0.076 & 0.37 & 0.51 & 0.84 & 1.4 & 1.2 \\
\hline
\end{tabular}

numbers are in $\%$ except the uncertainties on $t_{o}$; see text. 
TABLE 5

Expected uncertainties on fitted parameters for the range 1\%-60\% as a function of confidence level (vertically) and error on historical data (horizontally).

\begin{tabular}{ccccccc}
\hline Parameter: M & 1 & 5 & 10 & 15 & 20 & 25 \\
\hline 70 & 0.8 & 3.8 & 7.4 & 11.0 & 16.0 & 21.0 \\
75 & 1.1 & 4.1 & 8.1 & 13.0 & 18.0 & 24.0 \\
80 & 1.3 & 4.8 & 9.1 & 14.0 & 20.0 & 27.0 \\
85 & 1.4 & 5.5 & 10.0 & 16.0 & 23.0 & 30.0 \\
90 & 1.7 & 6.6 & 12.0 & 19.0 & 29.0 & 36.0 \\
95 & 2.4 & 7.9 & 15.0 & 21.0 & 44.0 & 44.0 \\
99 & 3.3 & 10.0 & 19.0 & 29.0 & 52.0 & 65.0 \\
\hline Parameter: $\alpha$ & 1 & 5 & 10 & 15 & 20 & 25 \\
\hline 70 & 0.3 & 1.5 & 3.0 & 4.3 & 6.2 & 7.9 \\
75 & 0.4 & 1.7 & 3.5 & 4.7 & 6.8 & 8.8 \\
80 & 0.4 & 1.9 & 3.8 & 5.2 & 7.5 & 9.8 \\
85 & 0.5 & 2.2 & 4.4 & 6.0 & 8.6 & 11.0 \\
90 & 0.7 & 2.4 & 4.8 & 6.7 & 9.6 & 12.0 \\
95 & 0.9 & 3.2 & 5.7 & 7.9 & 1.0 & 13.0 \\
99 & 1.2 & 4.3 & 7.7 & 11.0 & 14.0 & 17.0 \\
\hline Parameter: $t_{o}$ & 1 & 5 & 10 & 15 & 20 & 25 \\
\hline 70 & 0.016 & 0.072 & 0.15 & 0.19 & 0.29 & 0.39 \\
75 & 0.018 & 0.081 & 0.17 & 0.21 & 0.33 & 0.45 \\
80 & 0.022 & 0.091 & 0.18 & 0.26 & 0.37 & 0.49 \\
85 & 0.025 & 0.11 & 0.21 & 0.30 & 0.41 & 0.55 \\
90 & 0.029 & 0.13 & 0.24 & 0.34 & 0.51 & 0.67 \\
95 & 0.040 & 0.15 & 0.28 & 0.40 & 0.66 & 0.77 \\
99 & 0.060 & 0.19 & 0.36 & 0.50 & 0.84 & 0.96 \\
\hline
\end{tabular}

All numbers are in $\%$ except the uncertainties on $t_{o}$; see text.

TABLE 6

Expected uncertainties on fitted parameters for the range 1\%-70\% as a function of confidence level (vertically) and error on historical data (horizontally).

\begin{tabular}{ccccccc}
\hline Parameter: M & 1 & 5 & 10 & 15 & 20 & 25 \\
\hline 70 & 0.8 & 2.5 & 5.1 & 9.2 & 11.0 & 14.0 \\
75 & 0.9 & 2.8 & 5.6 & 10.0 & 12.0 & 16.0 \\
80 & 1.0 & 3.4 & 6.6 & 11.0 & 13.0 & 18.0 \\
85 & 1.2 & 3.8 & 7.5 & 13.0 & 15.0 & 20.0 \\
90 & 1.5 & 4.3 & 8.5 & 14.0 & 16.0 & 22.0 \\
95 & 1.9 & 5.6 & 9.8 & 16.0 & 20.0 & 25.0 \\
99 & 2.8 & 7.5 & 15.0 & 21.0 & 28.0 & 30.0 \\
\hline Parameter: $\alpha$ & 1 & 5 & 10 & 15 & 20 & 25 \\
\hline 70 & 0.3 & 1.5 & 2.7 & 4.1 & 5.4 & 6.9 \\
75 & 0.4 & 1.6 & 3.0 & 4.4 & 6.1 & 7.6 \\
80 & 0.5 & 1.7 & 3.3 & 5.1 & 6.7 & 8.5 \\
85 & 0.6 & 2.0 & 3.8 & 5.8 & 7.7 & 9.6 \\
90 & 0.7 & 2.3 & 4.4 & 6.7 & 8.7 & 11.0 \\
95 & 0.9 & 2.8 & 5.0 & 7.9 & 11.0 & 12.0 \\
99 & 1.2 & 3.6 & 6.7 & 11.0 & 14.0 & 16.0 \\
\hline Parameter: $t_{o}$ & 1 & 5 & 10 & 15 & 20 & 25 \\
\hline 70 & 0.015 & 0.052 & 0.11 & 0.17 & 0.22 & 0.28 \\
75 & 0.017 & 0.059 & 0.12 & 0.19 & 0.25 & 0.32 \\
80 & 0.030 & 0.073 & 0.13 & 0.21 & 0.29 & 0.36 \\
85 & 0.023 & 0.084 & 0.15 & 0.25 & 0.31 & 0.40 \\
90 & 0.027 & 0.094 & 0.17 & 0.38 & 0.35 & 0.44 \\
95 & 0.040 & 0.11 & 0.21 & 0.32 & 0.42 & 0.50 \\
99 & 0.058 & 0.16 & 0.28 & 0.46 & 0.53 & 0.66 \\
\hline
\end{tabular}

All numbers are in $\%$ except the uncertainties on $t_{o}$; see text. 
TABLE 7

Expected uncertainties on fitted parameters for the range $1 \%-80 \%$ as a function of confidence level (vertically) and error on historical data (horizontally).

\begin{tabular}{ccccccc}
\hline Parameter: M & 1 & 5 & 10 & 15 & 20 & 25 \\
\hline 70 & 0.5 & 1.9 & 3.9 & 5.1 & 8.1 & 8.9 \\
75 & 0.6 & 2.1 & 4.4 & 5.5 & 9.0 & 9.6 \\
80 & 0.7 & 2.4 & 4.8 & 6.2 & 9.8 & 11.0 \\
85 & 0.8 & 2.8 & 5.5 & 7.1 & 12.0 & 13.0 \\
90 & 1.1 & 3.3 & 6.3 & 9.1 & 13.0 & 16.0 \\
95 & 1.3 & 4.0 & 7.6 & 11.0 & 16.0 & 18.0 \\
99 & 2.2 & 5.6 & 9.1 & 15.0 & 21.0 & 31.0 \\
\hline Parameter: $\alpha$ & 1 & 5 & 10 & 15 & 20 & 25 \\
\hline 70 & 0.3 & 1.3 & 2.4 & 3.7 & 5.4 & 5.9 \\
75 & 0.3 & 1.4 & 2.6 & 4.2 & 5.8 & 6.4 \\
80 & 0.5 & 1.6 & 3.0 & 4.6 & 6.4 & 7.1 \\
85 & 0.5 & 1.7 & 3.4 & 5.0 & 7.1 & 7.9 \\
90 & 0.6 & 2.0 & 3.9 & 5.9 & 8.3 & 8.7 \\
95 & 0.8 & 2.4 & 4.7 & 7.5 & 9.9 & 10.0 \\
99 & 1.2 & 3.4 & 5.6 & 8.7 & 12.0 & 15.0 \\
\hline Parameter: $t_{0}$ & 1 & 5 & 10 & 15 & 20 & 25 \\
\hline 70 & 0.011 & 0.042 & 0.080 & 0.12 & 0.18 & 0.18 \\
75 & 0.013 & 0.048 & 0.090 & 0.13 & 0.19 & 0.21 \\
80 & 0.014 & 0.053 & 0.099 & 0.15 & 0.22 & 0.24 \\
85 & 0.017 & 0.059 & 0.11 & 0.16 & 0.25 & 0.28 \\
90 & 0.023 & 0.067 & 0.13 & 0.19 & 0.28 & 0.32 \\
95 & 0.029 & 0.083 & 0.15 & 0.22 & 0.34 & 0.36 \\
99 & 0.056 & 0.12 & 0.19 & 0.29 & 0.40 & 0.54 \\
\hline
\end{tabular}

All numbers are in $\%$ except the uncertainties on $t_{o}$; see text.

TABLE 8

Expected uncertainties on fitted parameters for the range 1\%-90\% as a function of confidence level (vertically) and error on historical data (horizontally).

\begin{tabular}{ccccccc}
\hline Parameter: M & 1 & 5 & 10 & 15 & 20 & 25 \\
\hline 70 & 0.3 & 1.4 & 2.9 & 4.2 & 6.0 & 7.1 \\
75 & 0.4 & 1.5 & 3.2 & 4.6 & 7.1 & 7.8 \\
80 & 0.5 & 1.9 & 3.5 & 5.2 & 8.1 & 8.5 \\
85 & 0.5 & 2.2 & 4.0 & 6.1 & 8.7 & 9.9 \\
90 & 0.6 & 2.5 & 4.7 & 7.0 & 10.0 & 11.0 \\
95 & 0.9 & 3.2 & 5.8 & 8.6 & 12.0 & 14.0 \\
99 & 1.5 & 4.6 & 8.6 & 12.0 & 16.0 & 20.0 \\
\hline Parameter: $\alpha$ & 1 & 5 & 10 & 15 & 20 & 25 \\
\hline 70 & 0.2 & 1.2 & 2.3 & 3.4 & 4.7 & 5.4 \\
75 & 0.3 & 1.3 & 2.5 & 3.8 & 5.1 & 6.1 \\
80 & 0.3 & 1.4 & 2.8 & 4.4 & 5.6 & 7.0 \\
85 & 0.4 & 1.5 & 3.1 & 4.9 & 6.0 & 7.7 \\
90 & 0.5 & 1.9 & 3.5 & 5.6 & 7.0 & 8.4 \\
95 & 0.6 & 2.2 & 4.1 & 6.3 & 8.3 & 9.9 \\
99 & 1.0 & 3.3 & 5.4 & 8.6 & 10.0 & 14.0 \\
\hline Parameter: $t_{o}$ & 1 & 5 & 10 & 15 & 20 & 25 \\
\hline 70 & 0.008 & 0.031 & 0.059 & 0.093 & 0.12 & 0.14 \\
75 & 0.009 & 0.034 & 0.067 & 0.10 & 0.14 & 0.16 \\
80 & 0.010 & 0.037 & 0.073 & 0.11 & 0.16 & 0.19 \\
85 & 0.013 & 0.044 & 0.083 & 0.12 & 0.17 & 0.21 \\
90 & 0.014 & 0.050 & 0.094 & 0.14 & 0.20 & 0.23 \\
95 & 0.018 & 0.064 & 0.11 & 0.17 & 0.24 & 0.28 \\
99 & 0.025 & 0.082 & 0.16 & 0.23 & 0.29 & 0.35 \\
\hline
\end{tabular}

All numbers are in \% except the uncertainties on $t_{o}$; see text. 
TABLE 9

Expected uncertainties on fitted parameters for the range 1\%-99\% as a function of confidence level (vertically) and error on historical data (horizontally).

\begin{tabular}{cccccc}
\hline Parameter: M & 5 & 10 & 15 & 20 & 25 \\
\hline 70 & 1.4 & 2.9 & 4.2 & 6.0 & 7.1 \\
75 & 1.5 & 3.2 & 4.6 & 7.0 & 7.8 \\
80 & 1.9 & 3.5 & 5.2 & 7.8 & 8.5 \\
85 & 2.2 & 4.0 & 6.1 & 8.7 & 9.9 \\
90 & 2.5 & 4.7 & 7.0 & 9.7 & 11.0 \\
95 & 3.2 & 5.8 & 8.6 & 11.0 & 14.0 \\
99 & 4.4 & 8.6 & 12.0 & 16.0 & 18.0 \\
\hline Parameter: $\alpha$ & 5 & 10 & 15 & 20 & 25 \\
\hline 70 & 0.9 & 1.9 & 2.9 & 3.7 & 4.7 \\
75 & 1.1 & 2.1 & 3.3 & 4.2 & 5.0 \\
80 & 1.2 & 2.3 & 3.5 & 4.6 & 5.5 \\
85 & 1.4 & 2.7 & 3.9 & 5.2 & 6.1 \\
90 & 1.5 & 3.0 & 4.4 & 6.0 & 7.0 \\
95 & 2.0 & 3.7 & 5.4 & 7.1 & 8.1 \\
99 & 2.6 & 5.1 & 7.1 & 9.5 & 10.0 \\
\hline Parameter: $t_{0}$ & 5 & 10 & 15 & 20 & 25 \\
\hline 70 & 0.020 & 0.041 & 0.058 & 0.081 & 0.11 \\
75 & 0.022 & 0.045 & 0.064 & 0.089 & 0.12 \\
80 & 0.025 & 0.049 & 0.071 & 0.098 & 0.13 \\
85 & 0.028 & 0.058 & 0.080 & 0.11 & 0.14 \\
90 & 0.034 & 0.064 & 0.089 & 0.13 & 0.16 \\
95 & 0.041 & 0.077 & 0.11 & 0.15 & 0.19 \\
99 & 0.057 & 0.096 & 0.14 & 0.21 & 0.24 \\
\hline
\end{tabular}

All numbers are in $\%$ except the uncertainties on $t_{o}$; see text.

$q\left(t_{i}\right)$ in the range of $1 \%$ to $40 \%$ of $\mathrm{M}$

$\cdots \cdots$.

$q\left(t_{i}\right)$ in the range of $1 \%$ to $90 \%$ of $\mathrm{M}$

$q\left(t_{i}\right)$ in the range of $1 \%$ to $99 \%$ of $\mathrm{M}$

For each time bin, statistical fluctuations were superimposed to simulate the inherent uncertainties on historical data. These fluctuations were generated according to a normal distribution around the theoretical value, with $\sigma$ varying flatly between $0 \%$ and $30 \%$. That is, $\check{\mathrm{g}}_{i}=q_{i}+\varepsilon q_{i}$ where $q_{i}$ the theoretical value for time bin i from equation (3) and $\varepsilon$ a Gaussian $\mathfrak{T}(0, \sigma)$ with zero the average and $\sigma$ the standard deviation.

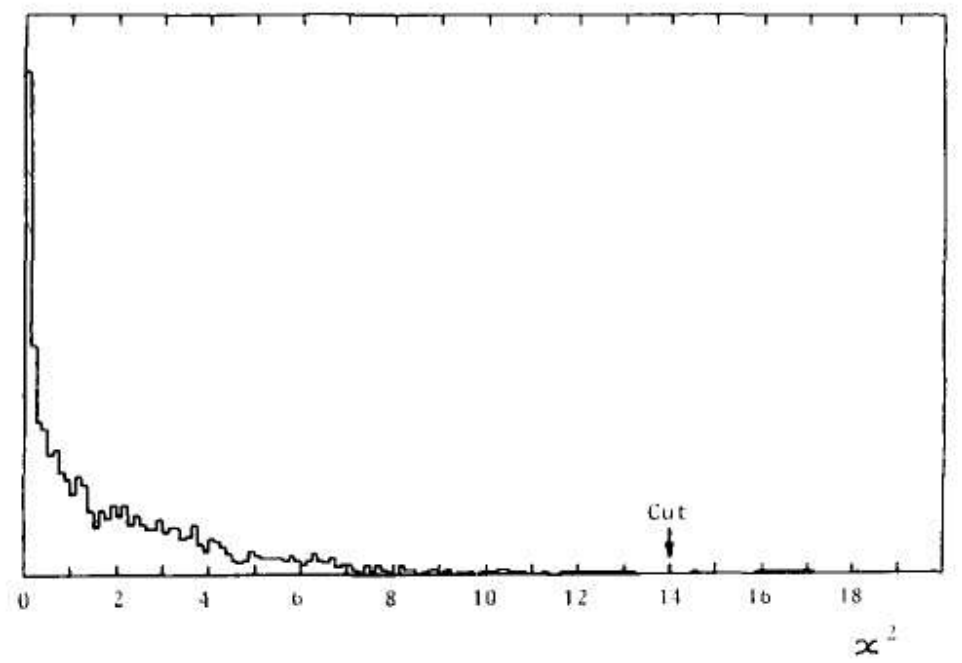

Fig. 2. $\chi^{2}$ distribution, equation (2), for the range $1 \%-50 \%$. The cut applied is shown. 


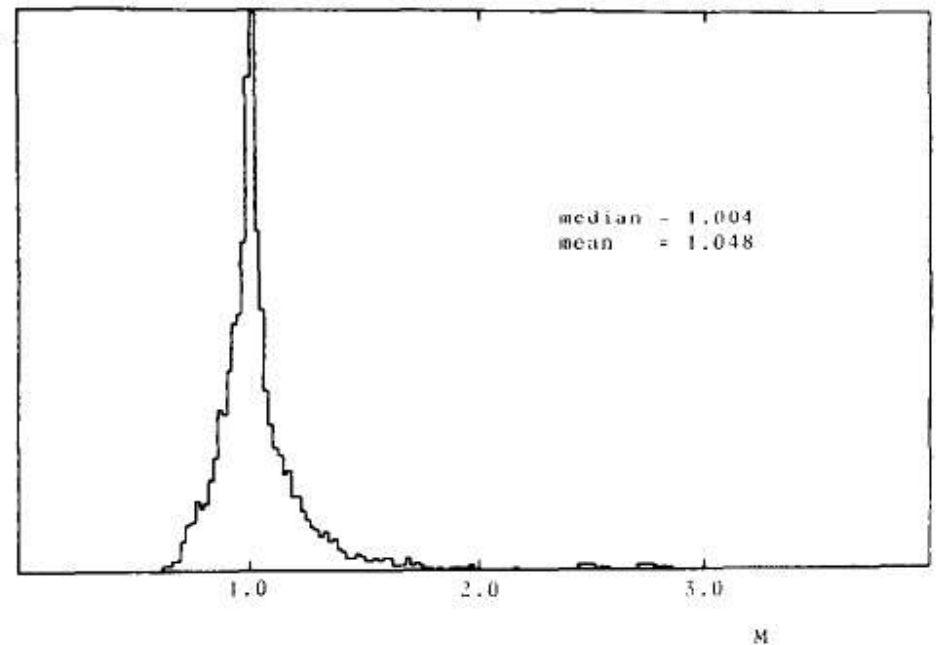

(a)

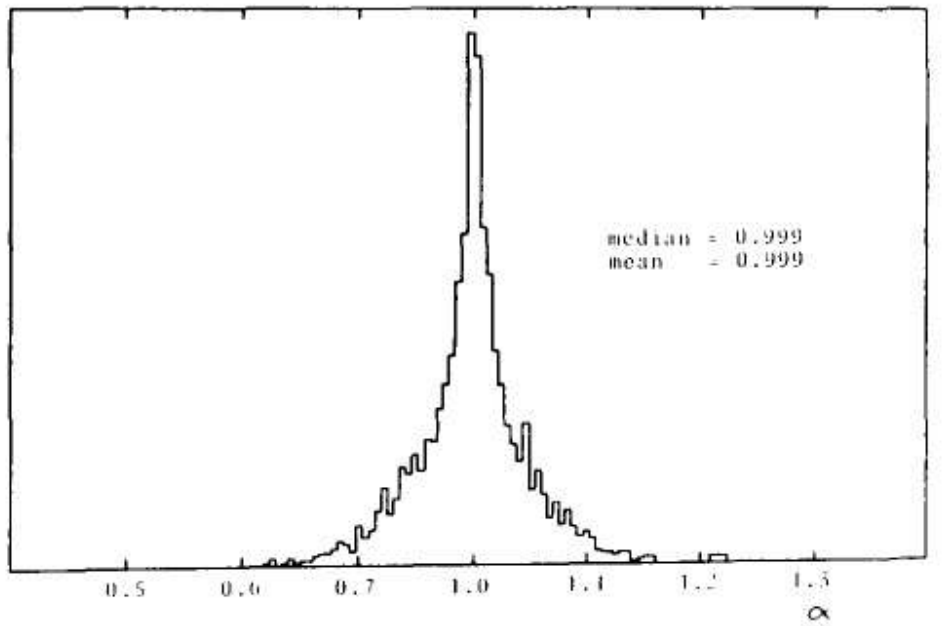

Fig. 3. Distributions for the fitted parameters in the range $1 \%$ $50 \%$.

(a) The potential $\mathrm{M}$, (b) the slope $\alpha$, (c) the time constant $t_{o}$.

(b)

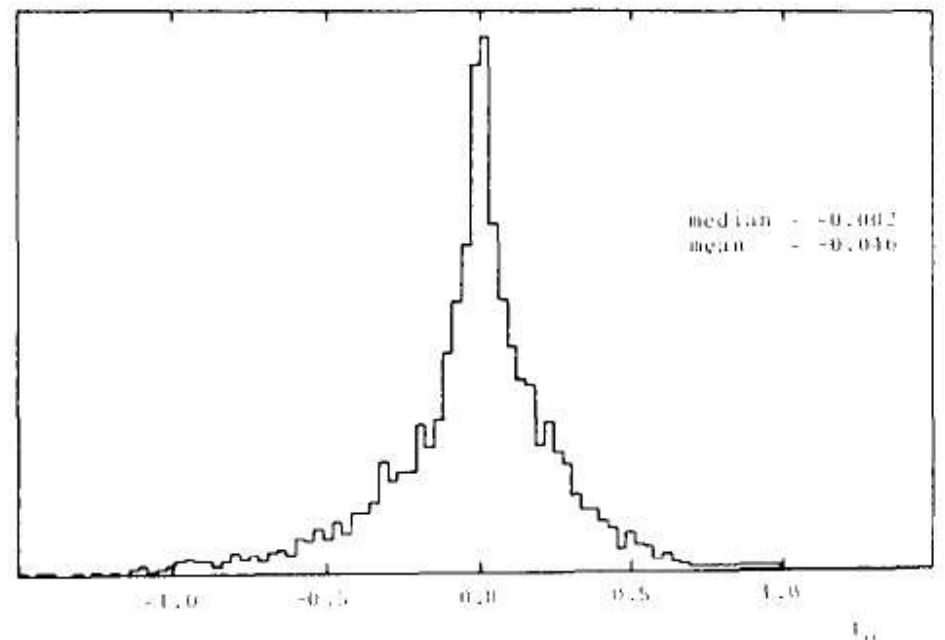

(c) 


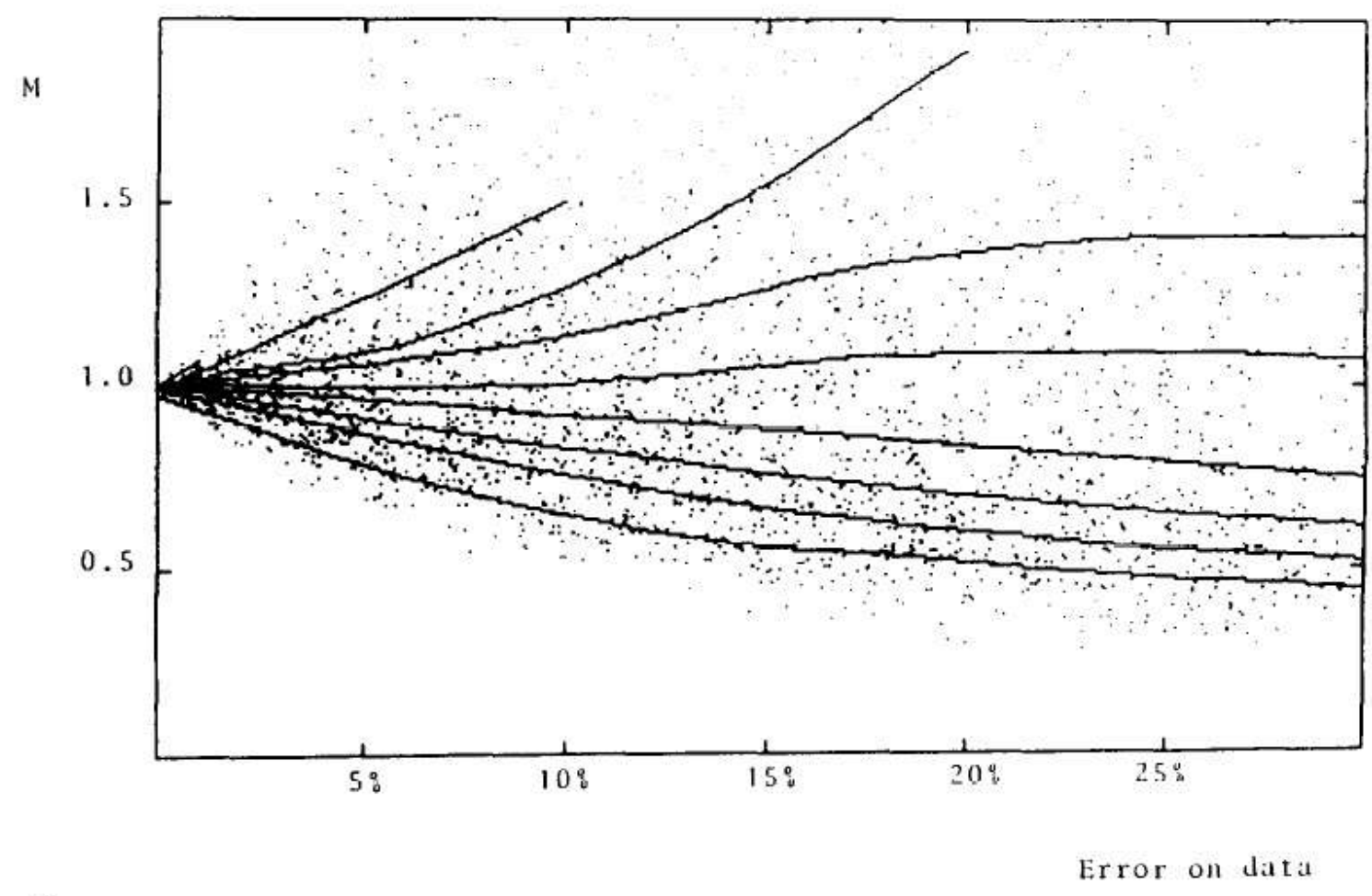

(a)

The life cycle curves thus obtained were subsequently integrated, producing the S-curve sections to be fitted. In this way we ensured that the statistically independent errors introduced on each time bin would be correctly accounted for in the cumulated S-curve representation.

A total of 33,693 different sections of S-curves were generated in this manner, evenly spread among the nine different time span ranges. The fits that followed were carried out by minimizing the $\chi^{2}$ of equation (2). A function minimization software package called MINUIT and developed at CERN [6] was used, providing values for $\mathrm{M}, \alpha$ and $t_{o}$ for each case. In addition the $\chi^{2}$ per degree of freedom was obtained.

\section{Results}

A typical $\chi^{2}$-distribution is shown in Figure 2, for the range $1 \%-50 \%$. The few very high values of $\chi^{2}$ on the tail are attributed to limitations of the function minimization software package for rare configurations of data points. A cut was applied, eliminating fits with very large $\chi^{2}$ and reducing the data sample by less than $1 \%$. The results presented below were minimally affected by this cut.

The parameters $\mathrm{M}, \alpha$ and $t_{o}$ recovered through the fits show no systematic deviation from the true values used in generating the data. Figure 3 shows typical distributions for the parameters of the range $1 \%-50 \%$. Even though a long tail on the $\mathrm{M}$ distribution biases the mean toward somewhat higher values, the median, which is more relevant in the determination of the confidence level, was found to be bias-free in all cases. Consequently, no systematic corrcctions arc neccssary to the fitted values.

For each range-nine in total - we present in tables I to IX the expected error on each of the parameters $\mathbf{M}, \alpha$ and $t_{o}$ as a function of the confidence level and statistical error of the data points. The expected error (EE) for a given parameter is defined as half the confidence interval, i.e., 


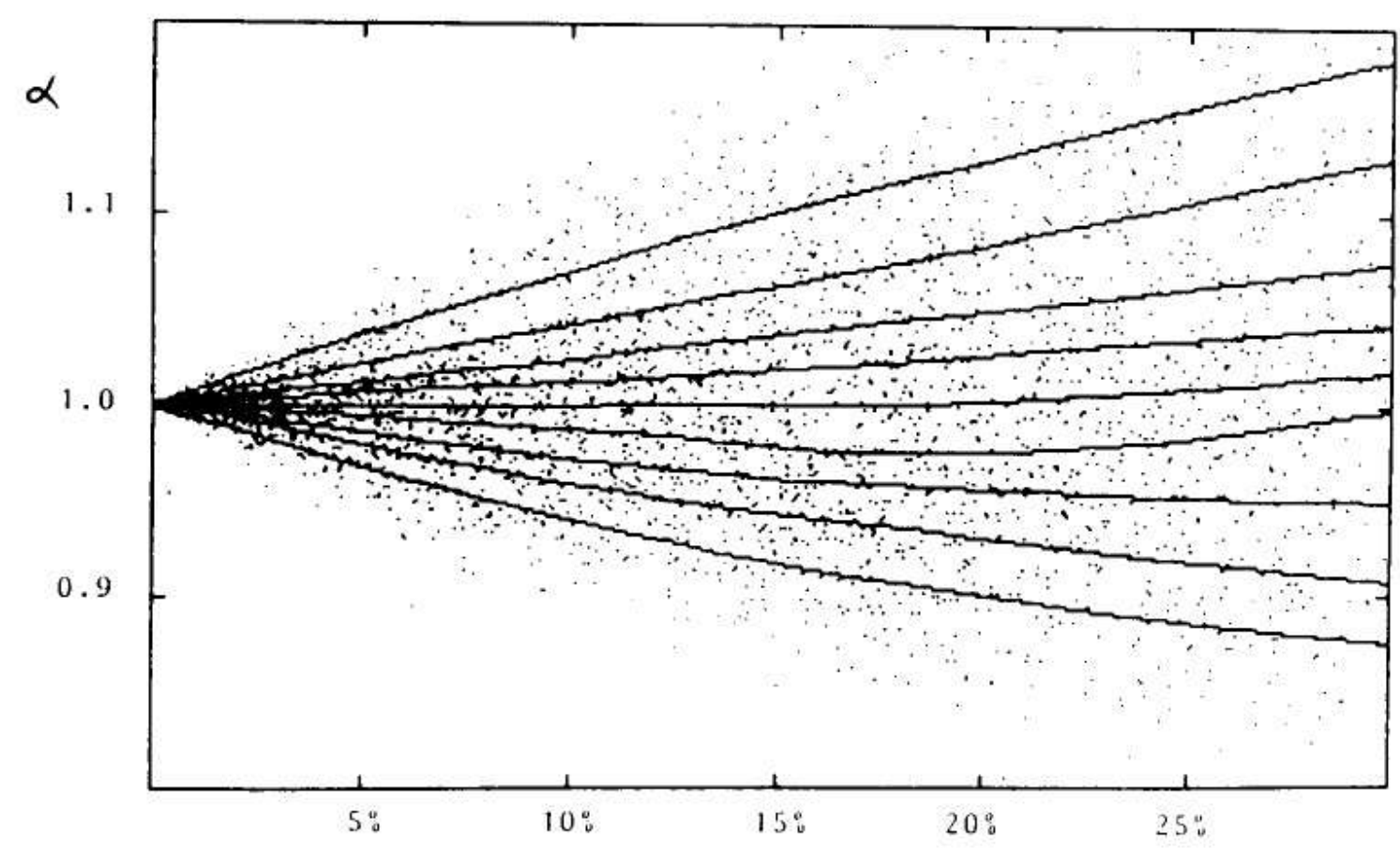

(b)

Lirror on data

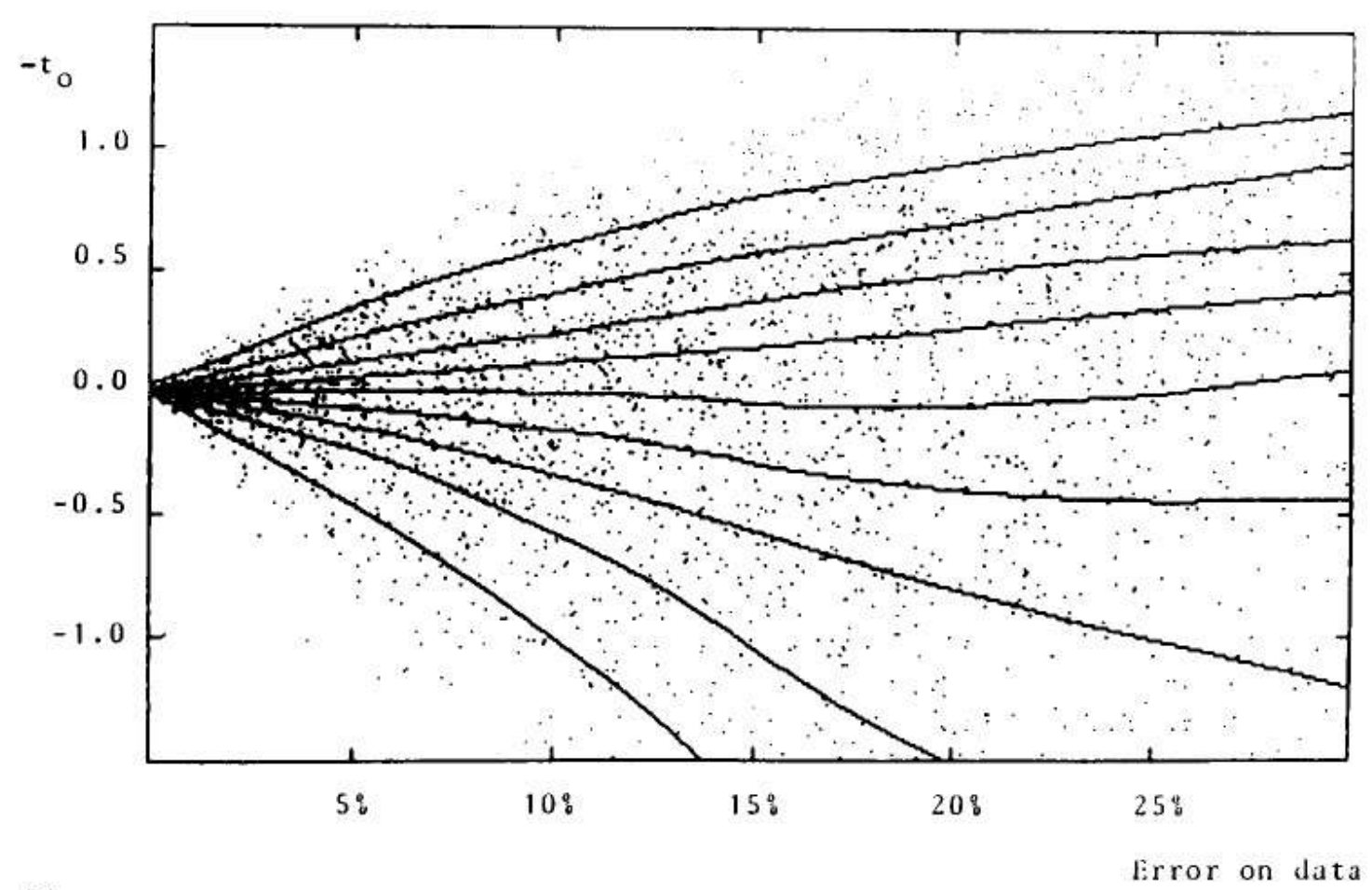

(c)

Fig. 4. Boundaries of $10 \%$ in the range $1 \%-20 \%$ as a function of error on the data for $M, a$ and $t_{o}$ in (a), (b) and (c) respectively; see text for explanations. 


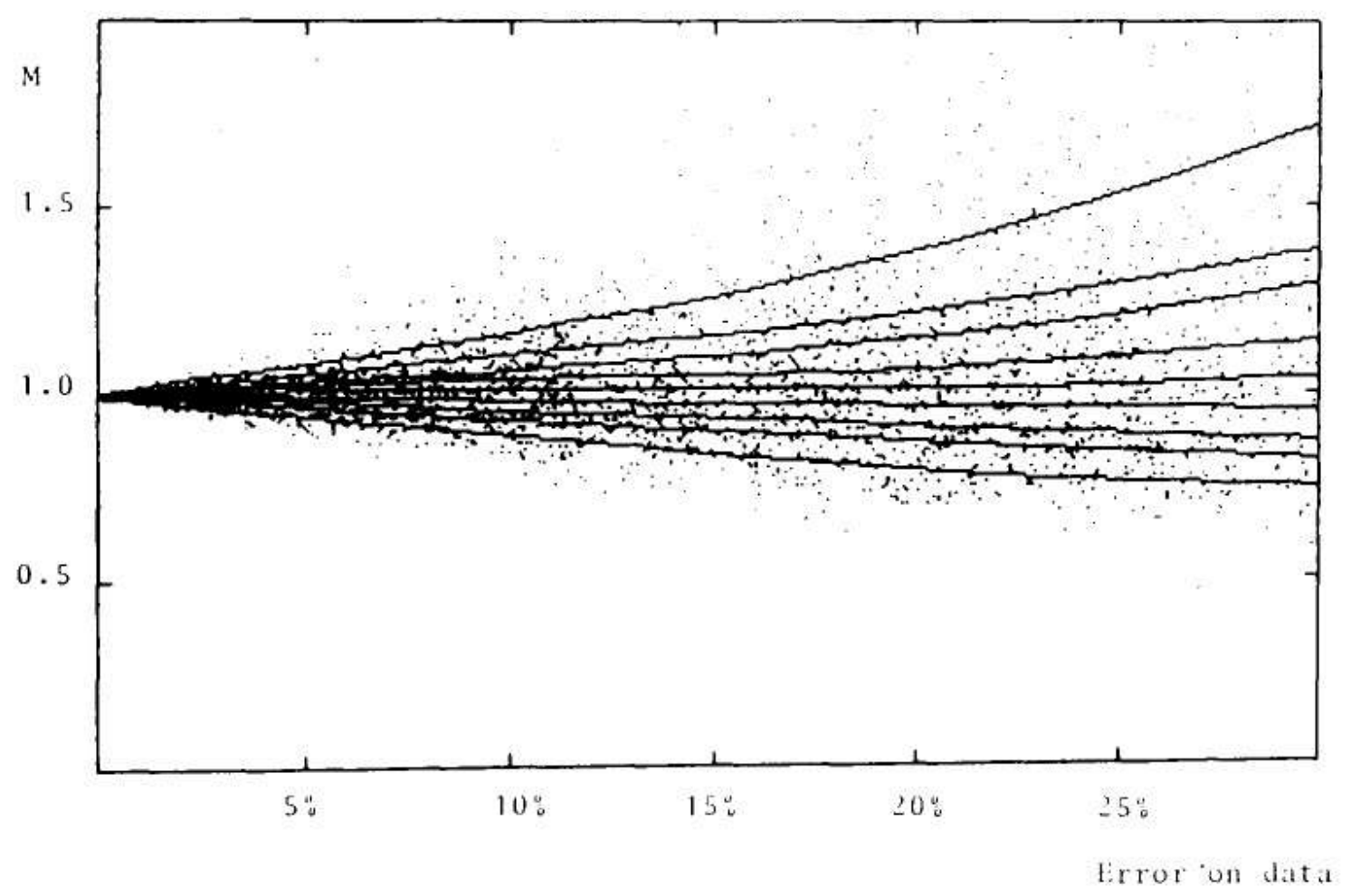

(a)

$$
\mathrm{EE}_{\mathrm{CL}}=(\mathrm{Max}-\mathrm{Min}) / 2
$$

so that the probability that the parameter value falls between Min and Max is equal to CL.

In the general case where $M$ and $\alpha$ are different than 1 , these errors correspond to percentages. The units of parameter $t_{o}$ are defined as: (total historical range)/20. Then the interpretation of these errors is the following: $\mathrm{M}_{\text {real }}=\mathrm{M}\left(1 \pm \mathrm{EE}_{\mathrm{CL}}\right)$ with confidence level CL.

Example: A fit on yearly historical data of supertanker construction gives $\mathbf{M}=115$. The historical period stops at 80 ships and we estimate an uncertainty on the reported yearly construction of $5 \%$. The range thus defined is $80 / 115=70 \%$. From table VI we obtain the uncertainty on $M$, namely $M=115 \pm 4.3 \%$ with $90 \%$ confidence level.

For complementary use and qualitative understanding, contour plots corresponding to Tables 1, 5 and 9 are also given in Figures 4 to 6 . For each parameter we indicate with dots the values obtained through the fits as a function of the uncertainty on the data. Solid lines are drawn in such a way as to contain $10 \%$ of the points between adjacent lines. The central line indicates the median. It was in this way that confidence levels were determined.

Finally, we address the question of correlations between the three parameters. The scatter plots of Figures 7,8 and 9 show typical cases some with evidence of a strong nonlinear correlation (Figure 7), while others show no visible correlation at all (Figure 9). Clearly, the larger the range, i.e., the bigger the fraction of the S-curve covered by the historical data, the more accurate the parameter determination and the less visible the correlation between the uncertainties of the parameters. Correlations become increasingly important as the range is reduced.

The presence of strong correlations allows the possibility of "what-if" scenarios to be carried out independently of the actual fits. From Figure 7(a), for example, we can see that for the same set of historical points, if $\alpha$ were to decrease by $5 \%$, passing from 


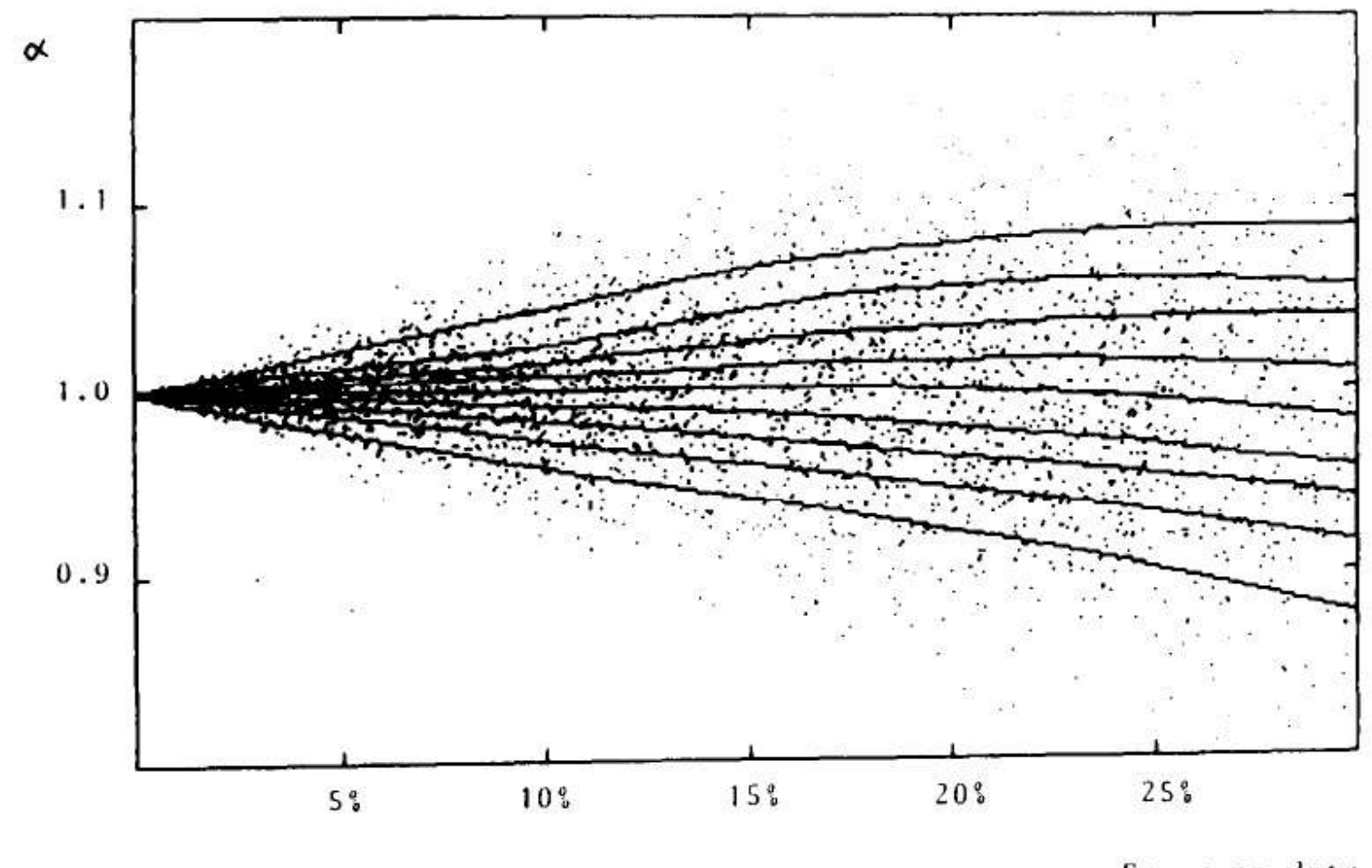

(b)

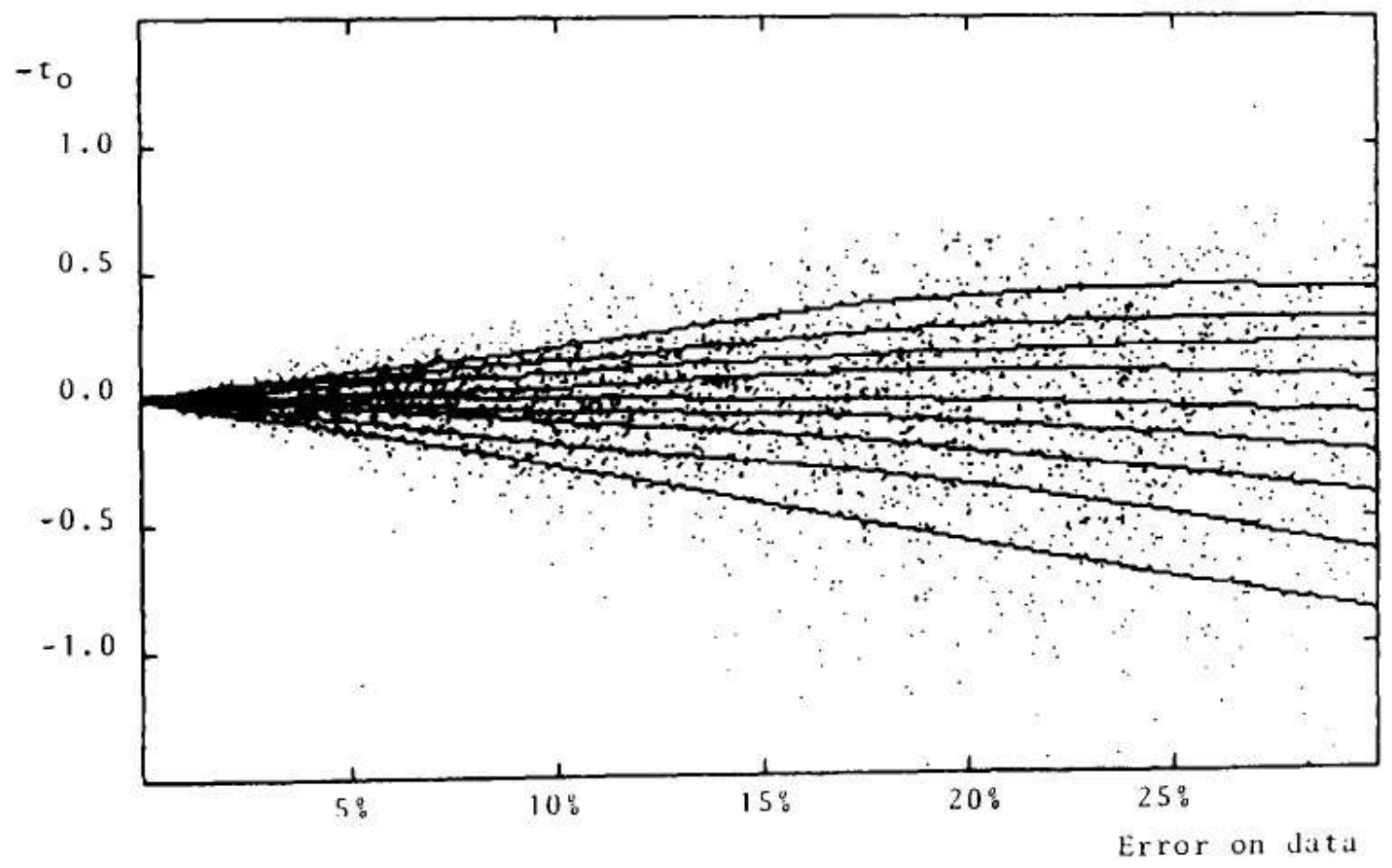

(c)

Fig. 5. Boundaries of $10 \%$ in the range $1 \%-50 \%$ as a function of error on the data for $M, \alpha$ and $t_{o}$ in (a), (b) and (c) respectively; see text for explanations. 


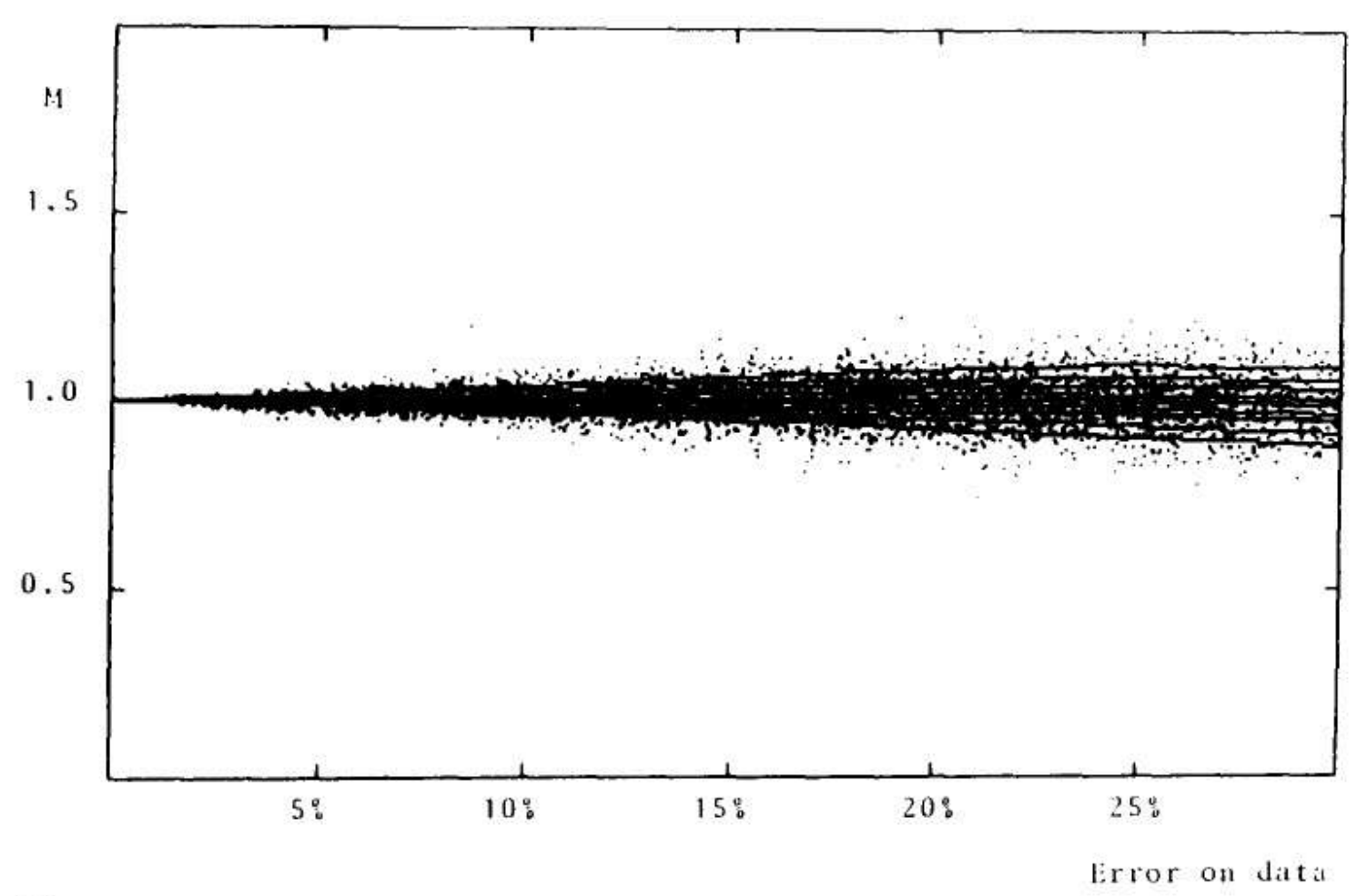

(a)

1.0 to 0.95 , the corresponding increase in $\mathrm{M}$ may be as high as $80 \%$, whereas a similar increase in $\alpha$ would result in a lesser decrease for $M$.

\section{Conclusions}

S-curve fitting can be meaningfully applied whenever the historical data cover a fair fraction (minimum of 20\%) of the full range of an S-curve. Points on the two extremities, i.e., below $\sim 5 \%$ or above $\sim 95 \%$ of the full range should not be expected to fit well. Minimization of a $\chi^{2}$ is a good approach to determine the three parameters defining the S-curve. Uncertainties for the values found for these parameters can be looked up in the tables and graphs provided in the previous section as a function of the error on the historical data and the desired confidence level.

Correlations between the uncertainties of the three parameters are important, the more so the smaller the available range of the S-curve under study. They can be used to play "what-if" games without having to do the fits, as long as it is not too late (the case of an almost complete S-curve). For example, they may offer an explanation as to the case of "child prodigies," so promising in their early life by their fast rate of growth but often disappointing by their modest final level.

The implication of these correlations can go very far. If we were to interfere with the growing process, e.g., decrease the rate of growth $\alpha$ - easily done in industry, hormonally done in biology - the probable effect would be an increase of $M$ and an increase of $t_{o}$ as long as, whatever the intervention, it was done "adiabatically" so as not to disturb the underlying logistic law. According to French folklore, "Qui veux voyager loin ménage sa monture," which means, "He who wants to travel far spares his horse."

In general, S-curve fitting, a natural and fundamental approach to forecasting, is more reliable than suspected. We can see for example from Table 4 that historical data covering the first half of the S-curve with estimated $10 \%$ errors per data point will yield a value for the final maximum, accurate to within $20 \%$ with $95 \%$ confidence level. This 


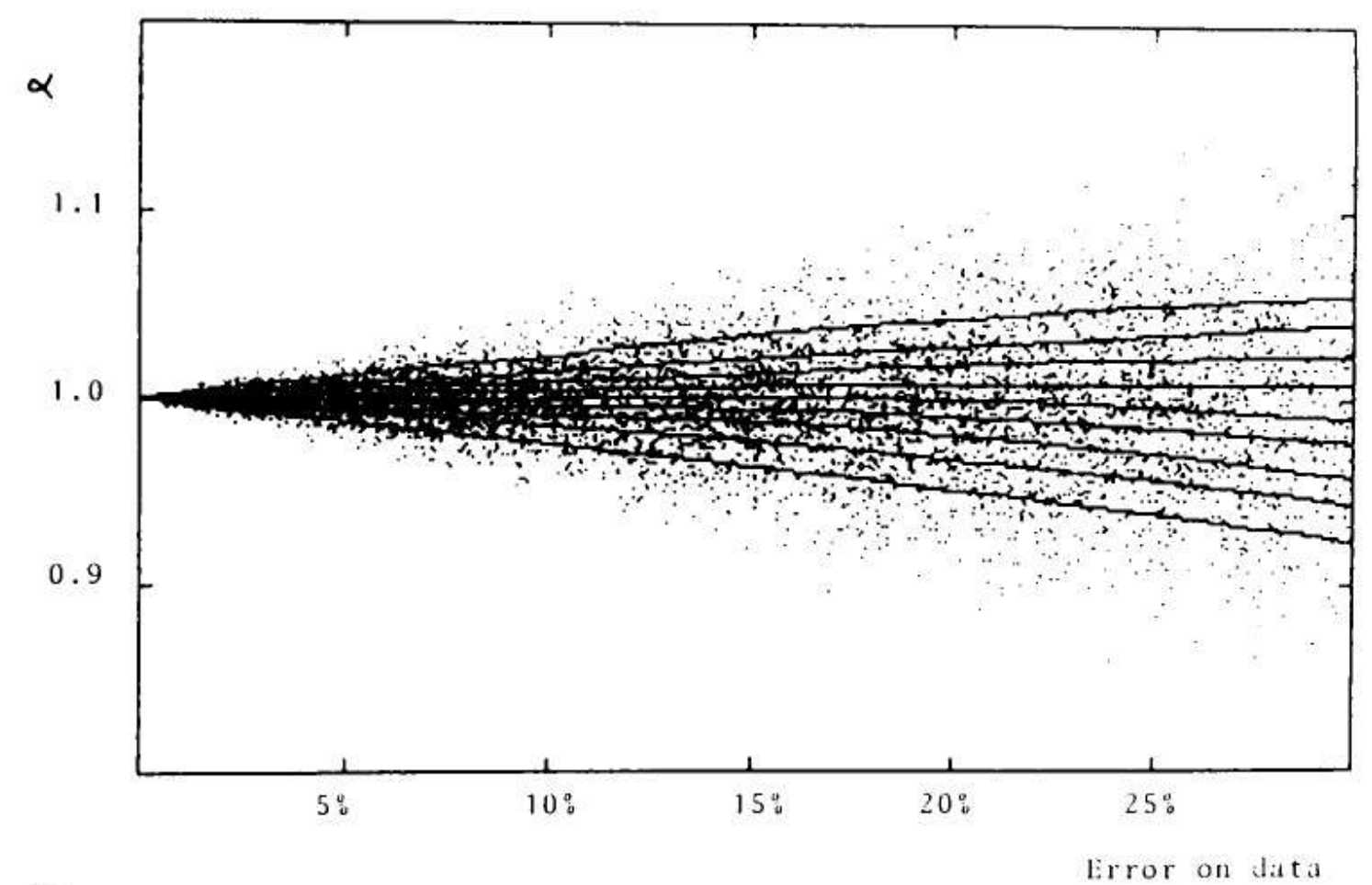

(b)

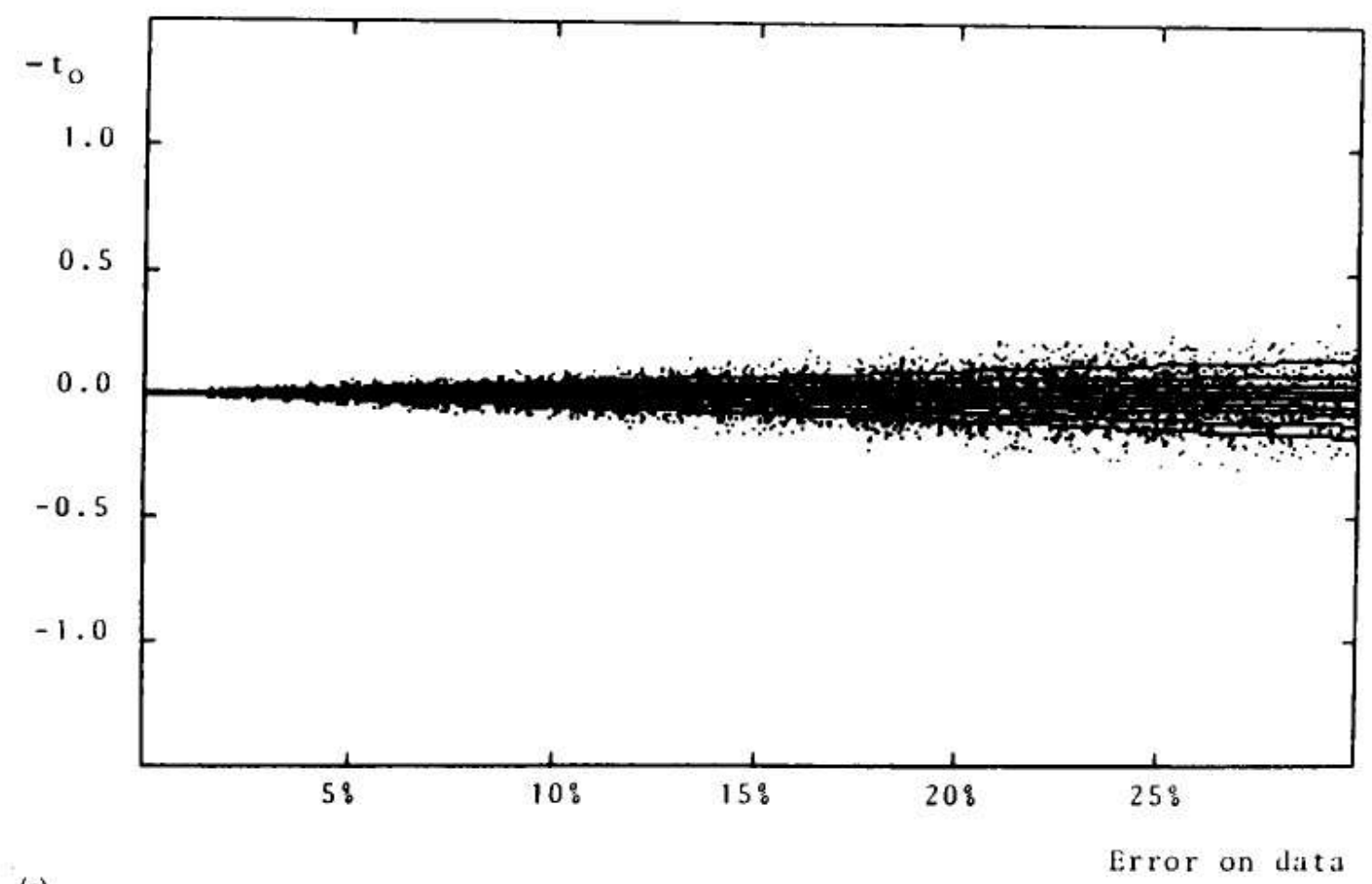

(c)

Fig. 6. Boundaries of $10 \%$ in the range $1 \%-99 \%$ as a function of error on the data for $M, \alpha$ and $t_{o}$ in (a), (b) and (c) respectively; see text for explanations. 


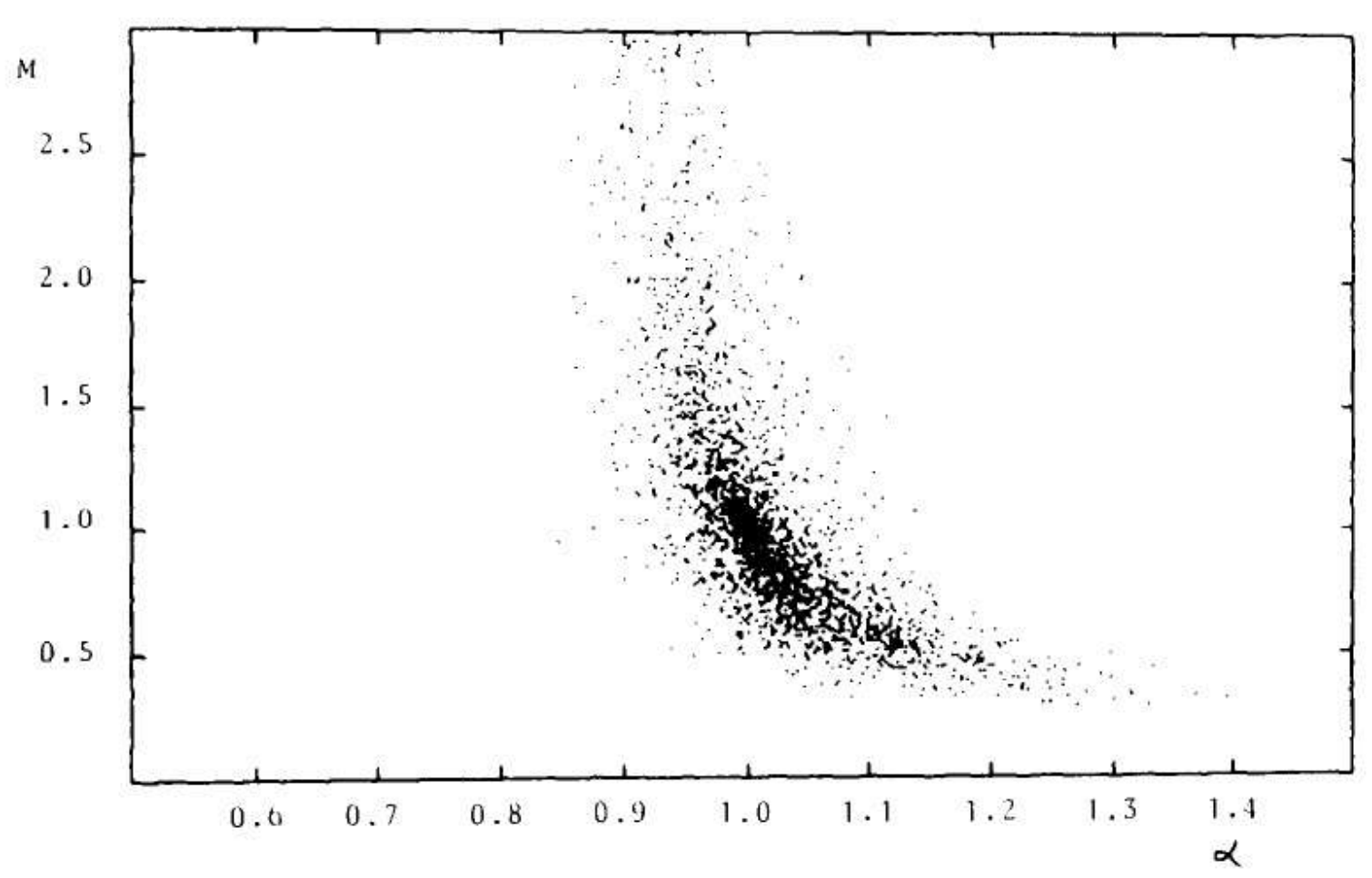

(a)

can be of invaluable importance when dealing with processes for which the final maximum has only been wildly speculated upon up to now.

\section{References}

1. Debecker, A., and Modis, T., Innovation among the Major Computer Manufacturers. Technological Forecasting and Social Change 33, 267-278 (1988).

2. Marchetti, C., Innovation, Industry and Economy: A Top-Down Analysis, International Institute for Applied Systems Analysis, A-2361, Laxemburg, Austria, pp. 83-86, December 1983.

3. Marchetti, C., Infrastructures for Movement, Technological Forecasting and Social Change 32, 373-393 (1987).

4. Fisher, J.C., and Pry, R.H., A Simple Substitution Model of Technological Change, Technological Forecasting and Social Change, 3, 75-88 (1971).

5. Narenda, S., et al., On the Voltera and Other Nonlinear Models of Interacting Populations, Reviews of Modern Physics, 43, 231-276, April 1971.

6. James, F., Minuit-Function Minimization, CERN, Geneva, Switzerland.

\section{Appendix}

\section{STATISTICS FOR LOGISTIC GROWTH}

Let $Q(t)$ be a random variable describing a population at time $t$. It follows that $Q(t)$ must be discrete and finite, limited by a final value $\mathrm{M}$, the niche capacity. Let $q(t)$ be an observed value of $Q(t)$. If the remaining $\mathrm{M}-q(t)$ individuals are equally likely, then the differential increment at time $t$, defined as

$$
(d Q(t)) d t=Q(t+d t)-Q(t)
$$

will obey a binominal law $B(\mathrm{M}-q(t), \lambda(t) d t)$

where $\lambda(t) d t$ is the probability of appearance. This probability is proportional to $d t$, and the proportionality coefficient, $\lambda(t)$, represents some kind of "fertility" or capability to fill the niche and is in general a function of time.

Knowing the probability law for $d Q(t)$ when $Q(t)=q(t)$, and assuming that $Q(t)$ obeys a binominal law $B(\mathrm{M}, f(t))$, we can show that $d Q(t)$ will obey the binominal 


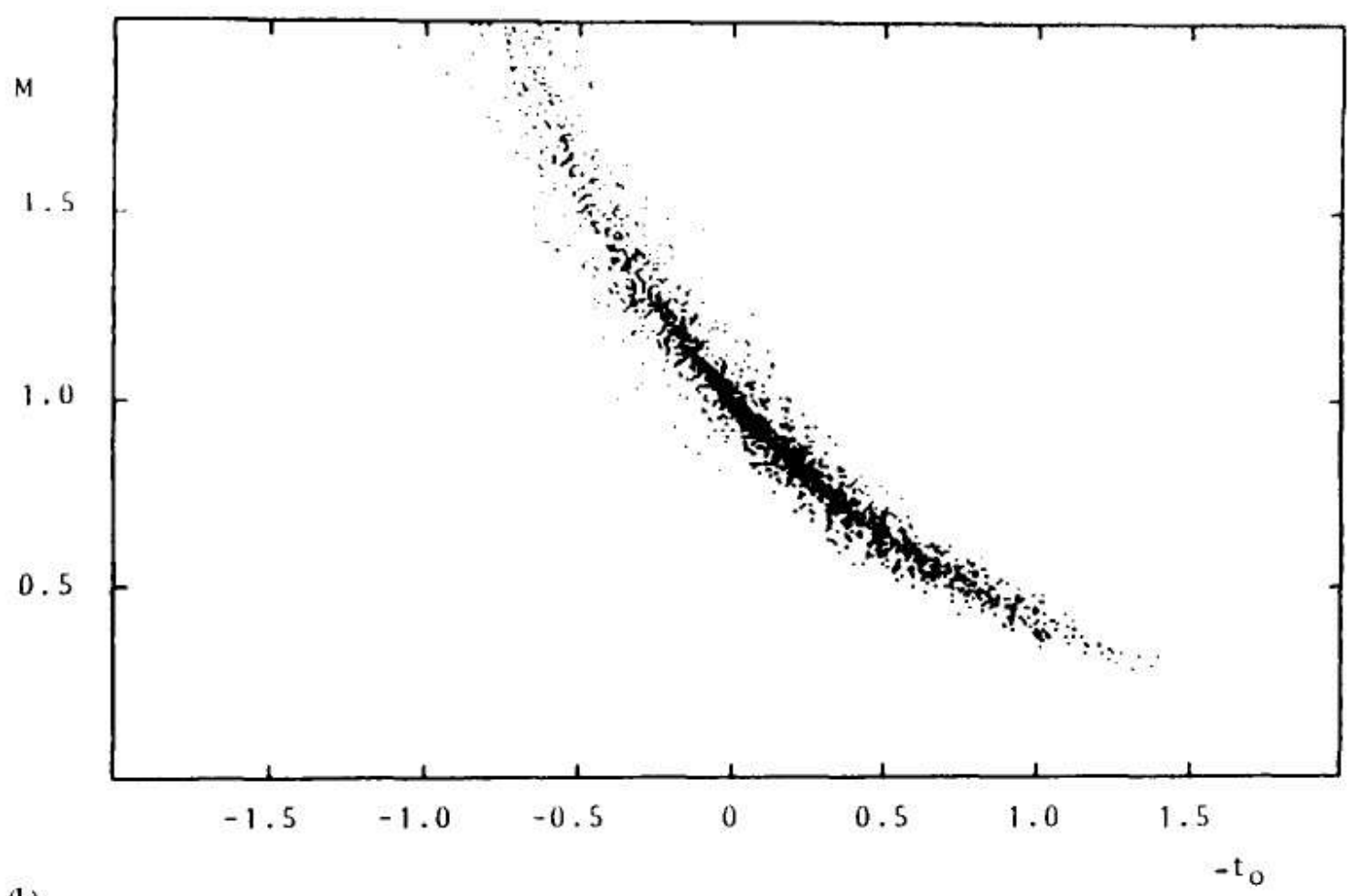

(b)

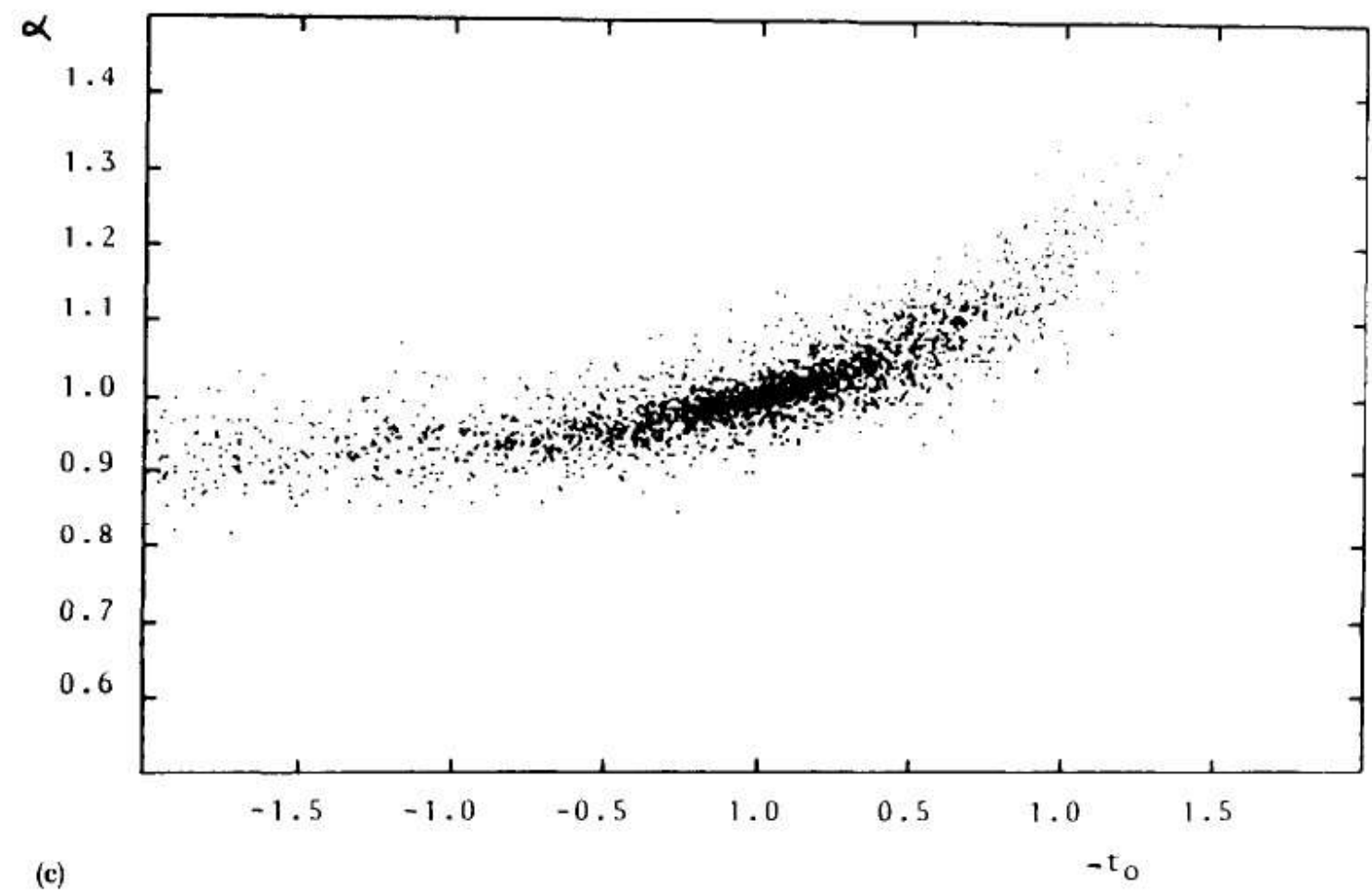

Fig. 7. Scatter plots for the range $1 \%-20 \%$ of one parameter against the other as determined by the fits. (a) $\mathrm{M}$ vs $\alpha$, (b) $\mathrm{M}$ vs $t_{\theta,}$ (c) $\alpha$ vs $t_{\theta}$. 


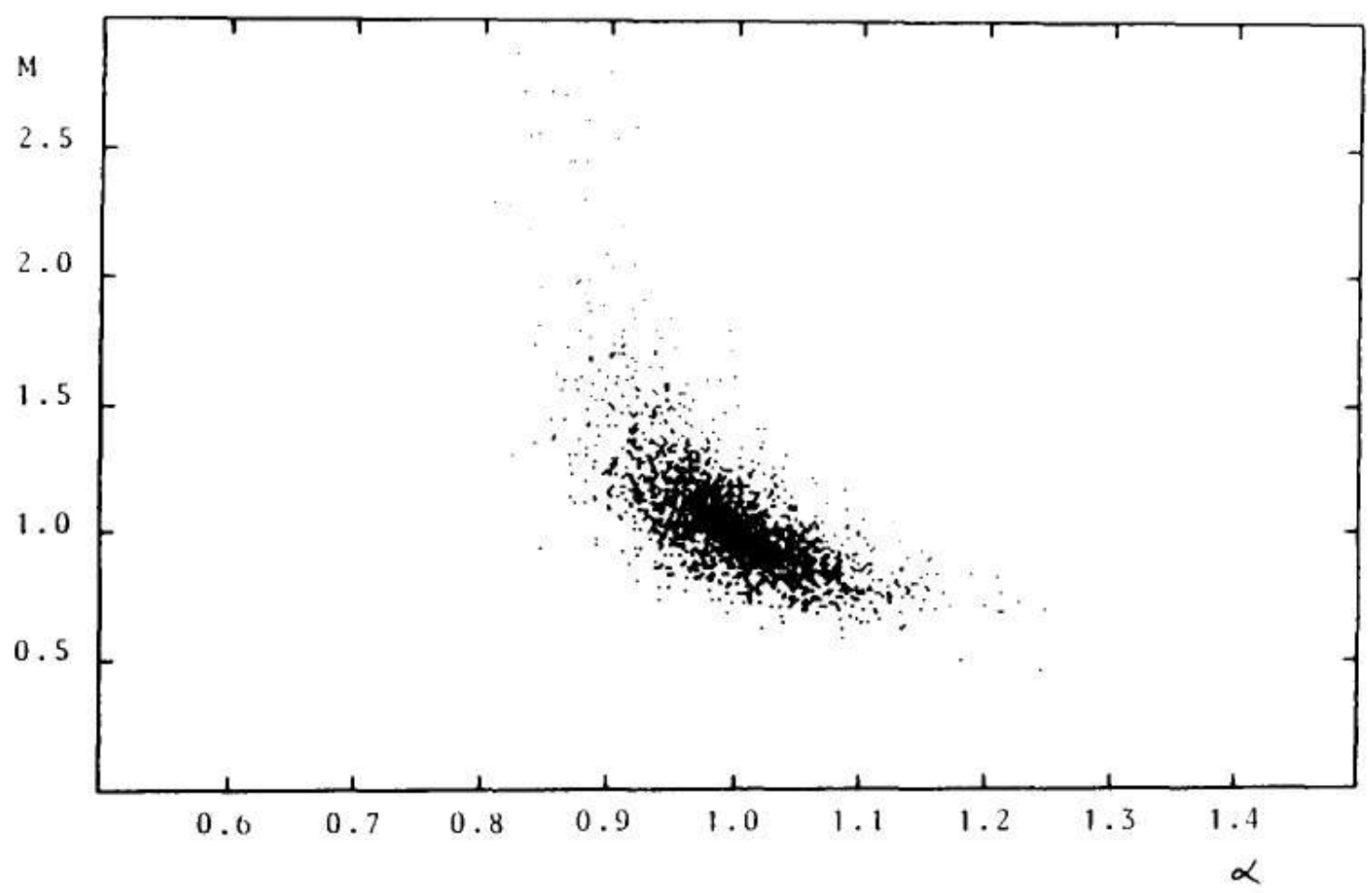

(a)

$\mathbb{B}(\mathrm{M}, \lambda(t)(1-f(t)) d t)$ and that $\mathrm{Q}(t+d t)=\mathrm{Q}(t)+d Q(t)$ will obey the binominal $B(\mathrm{M}, f(t+d t))$.

The parameter $f(t)=\mathrm{E}(Q(t)) / \mathrm{M}$ represents the expected fraction of the niche occupied at time $t$. It follows that $\mathrm{f}$ will be a solution of the differential equation

$$
d f=f(t+d t)-f(t)=\frac{\mathrm{E}(d Q)}{\mathrm{M}}=\lambda(1-f) d t
$$

For $\mathrm{t} \rightarrow-\infty, Q(t)$ becomes certain $(\mathrm{Q}(\mathrm{t})=0)$, thus binominal. The above inductive reasoning will then show that $\mathrm{Q}(\mathrm{t})$ is binominal everywhere and if we take the growth proportional to the size, namely $\lambda f(t)=\alpha f(t)$, then we arrive at the Voltera equation

$$
d f=f(1-f) d t
$$

with the solution

$$
f=\frac{1}{1+e^{-a\left(t-t_{o}\right)}}
$$

$t_{o}$ being an integration constant.

We then have for the expectation and variance of $\mathrm{Q}(t)$

$$
\mathrm{E}(\mathrm{Q}(t))=\mathrm{M} f=\frac{\mathrm{M}}{1+e^{-\mathrm{a}\left(t-t_{0}\right)}}
$$

and

$$
\sigma^{2}(\mathrm{Q}(t))=\mathrm{M} f(1-f)=\frac{\mathrm{M}}{\left(1+e^{\alpha\left(t-t_{o}\right)}\right)\left(1+e^{-\alpha\left(t-t_{o}\right)}\right)}
$$

if $M$ is large and $O \ll q(t) \ll M$, then $Q(t)$ is practically Gaussian and 


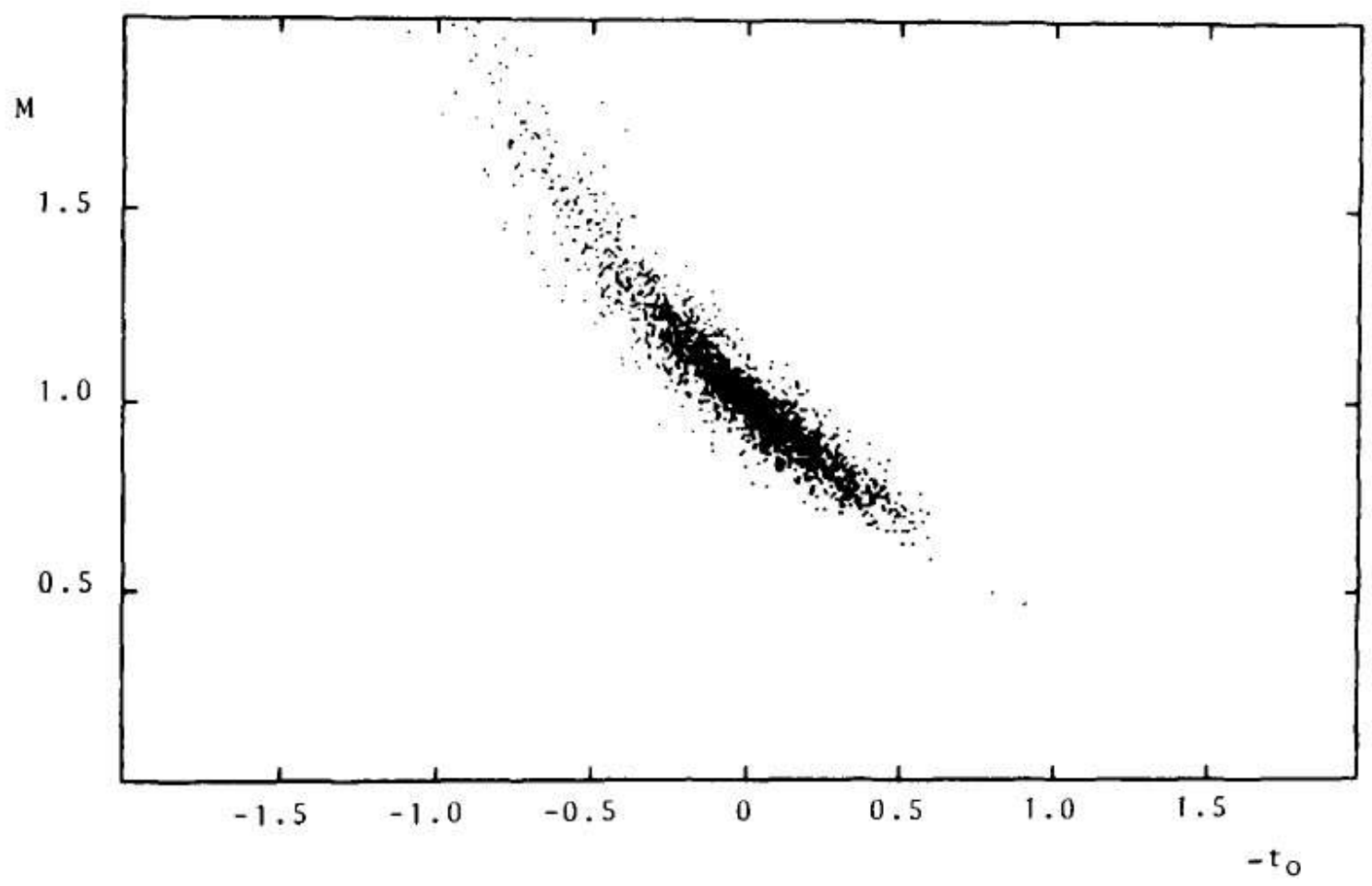

(b)

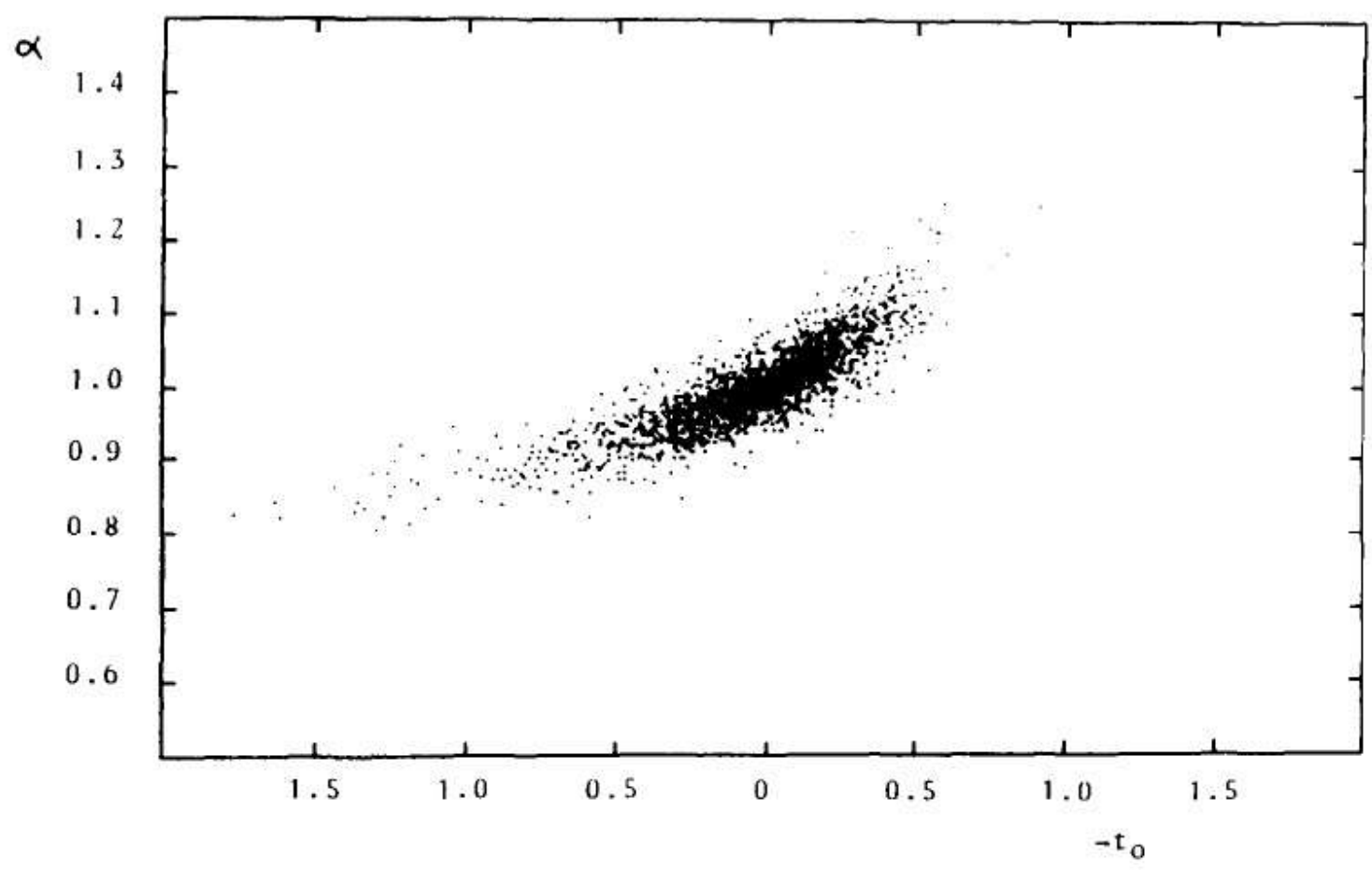

(c)

Fig. 8. Scatter plots for the range $1 \%-50 \%$ of one parameter against the other as determined by the fits. (a) $M$ vs $\alpha$, (b) $M$ vs $t_{0}$, (c) $\alpha$ vs $t_{o}$. 


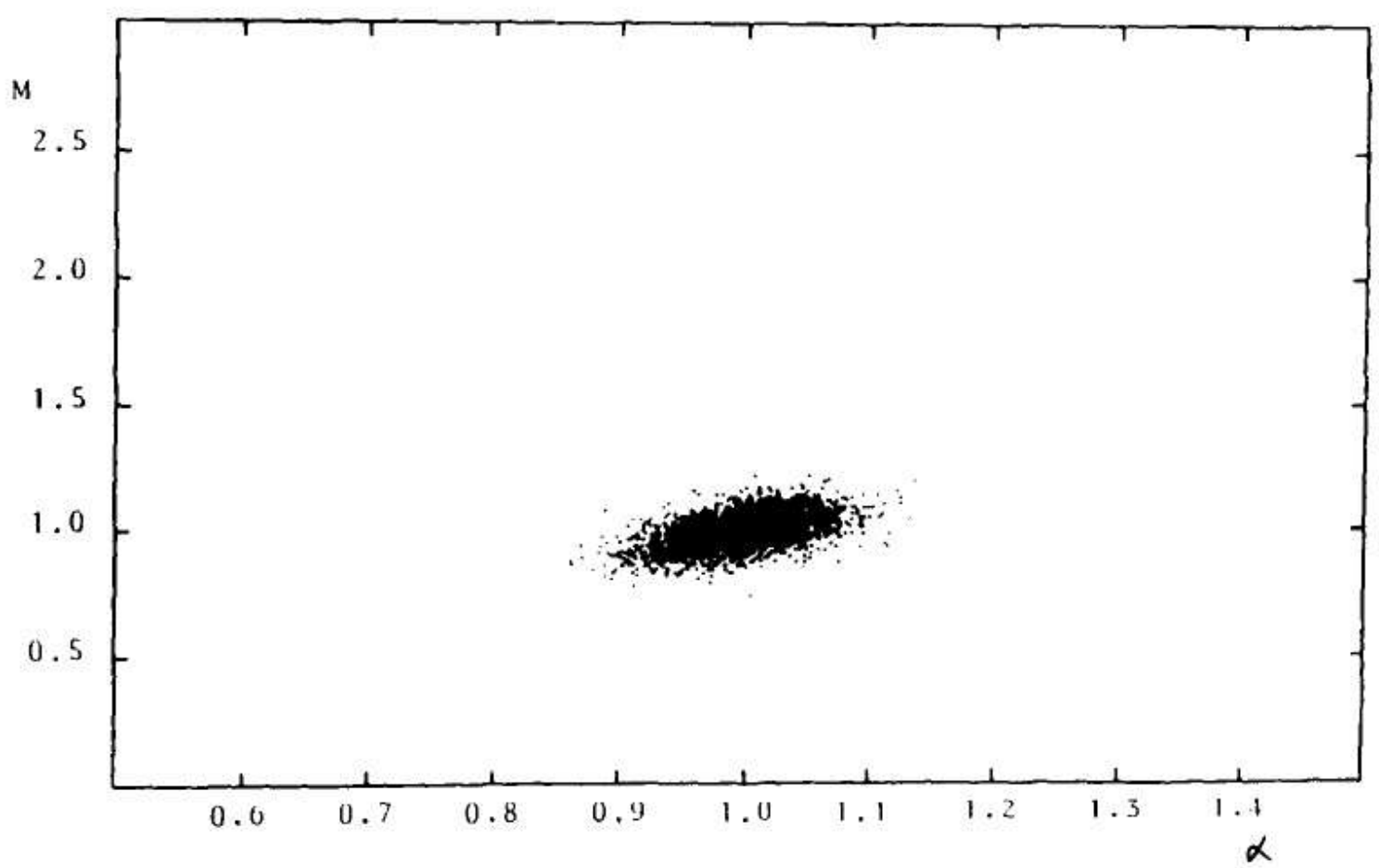

(a)

$$
\mathrm{U}=\sum_{i=1}^{n} \frac{\left(q\left(t_{i}\right)-\mathbf{M} f\left(t_{i}\right)\right)^{2}}{\mathrm{M} f\left(t_{i}\right)\left(1-f\left(t_{i}\right)\right)}
$$

obeys a $\chi^{2}$ distribution law with $\mathrm{n}-3$ degrees of freedom.

At the extremities, $Q(t)$ is distributed according to Poisson probability law rather than Gaussian, and the most probable observed value becomes $q=0$ or $q=\mathrm{M}$. Therefore, in order to stay within the Gaussian approximation, we must avoid small and large $f$. This is commonly applied as a $10 \%$ rule, i.e., excluding the regions of $f<10 \%$ or $f>90 \%$. 


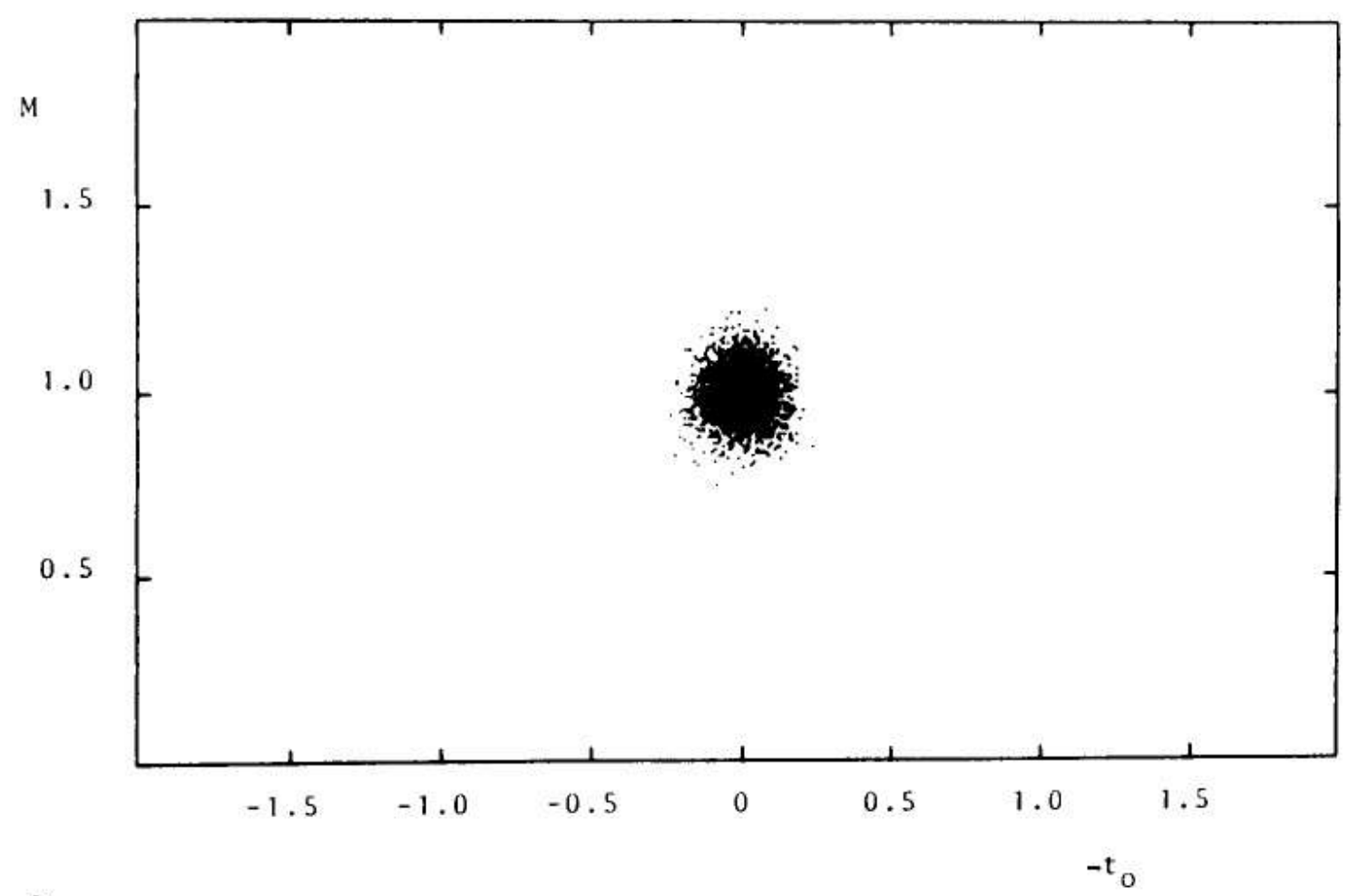

(b)

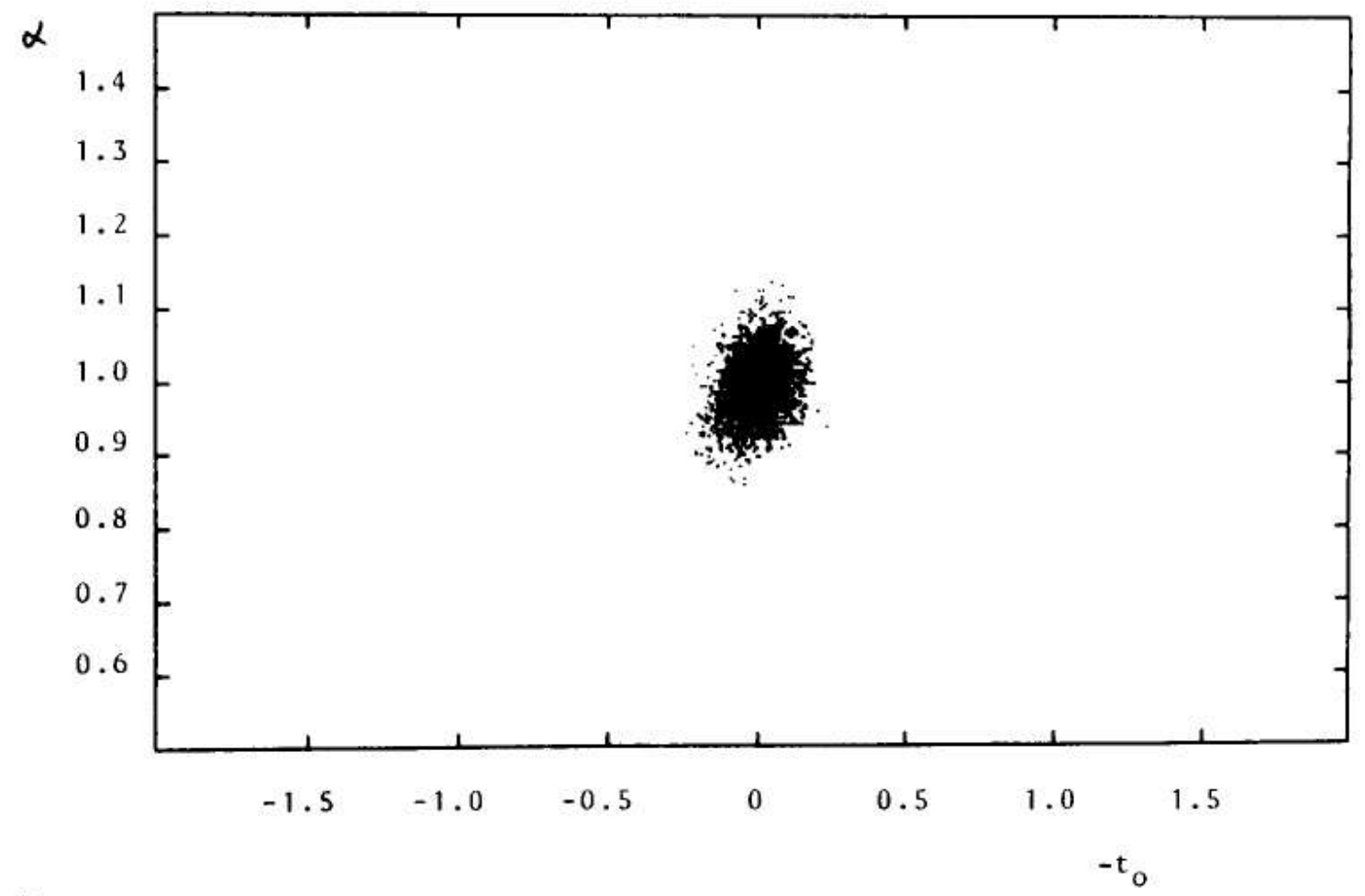

(c)

Fig. 9. Scatter plots for the range 1\%-99\% of one parameter against the other as determined by the fits. (a) $M$ vs $\alpha$, (b) $M$ vs $t_{o}$, (c) $\alpha$ vs $t_{o}$. 